Homology, Homotopy and Applications, vol.9(1), 2007, pp.295-329

\title{
ON THE HOMOTOPY TYPE AND THE FUNDAMENTAL CROSSED COMPLEX OF THE SKELETAL FILTRATION OF A CW-COMPLEX
}

\author{
JOÃO FARIA MARTINS
}

(communicated by Ronald Brown)

\begin{abstract}
We prove that if $M$ is a CW-complex, then the homotopy type of the skeletal filtration of $M$ does not depend on the cell decomposition of $M$ up to wedge products with $n$-disks $D^{n}$, when the latter are given their natural CW-decomposition with unique cells of order $0,(n-1)$ and $n$, a result resembling J.H.C. Whitehead's work on simple homotopy types. From the higher homotopy van Kampen Theorem (due to R. Brown and P.J. Higgins) follows an algebraic analogue for the fundamental crossed complex $\Pi(M)$ of the skeletal filtration of $M$, which thus depends only on the homotopy type of $M$ (as a space) up to free product with crossed complexes of the type $\mathcal{D}^{n} \doteq \Pi\left(D^{n}\right), n \in \mathbb{N}$. This expands an old result (due to J.H.C. Whitehead) asserting that the homotopy type of $\Pi(M)$ depends only on the homotopy type of $M$. We use these results to define a homotopy invariant $I_{\mathcal{A}}$ of $\mathrm{CW}$-complexes for each finite crossed complex $\mathcal{A}$. We interpret it in terms of the weak homotopy type of the function space $\operatorname{TOP}((M, *),(|\mathcal{A}|, *))$, where $|\mathcal{A}|$ is the classifying space of the crossed complex $\mathcal{A}$.
\end{abstract}

\section{Contents}

1 Introduction $\quad 296$

2 Preliminaries $\quad 298$

2.1 Cofibred filtrations . . . . . . . . . . . . . . . . . 298

2.2 Crossed complexes . . . . . . . . . . . . . . . . 302

3 The homotopy type of the skeletal filtration of a CW-complex 312

3.1 Dimension two . . . . . . . . . . . . . . . . 312

3.2 The general case . . . . . . . . . . . . . . . . . 314

Received April 7, 2006, revised January 12, 2007; published on March 6, 2007.

2000 Mathematics Subject Classification: 55P10, 55Q05, 57M27.

Key words and phrases: CW-complex, skeletal filtration, crossed complex, higher homotopy van Kampen Theorem, invariants of homotopy types.

Copyright (C) 2007, International Press. Permission to copy for private use granted. 
4 On the fundamental crossed complex of a CW-complex $\quad 321$

4.1 The dependence of $\Pi(M)$ on the cell decomposition of $M$. . . . . 321

4.2 A rational number valued homotopy invariant $I_{\mathcal{A}}$. . . . . . . . . . . 322

\section{Introduction}

The concept of a crossed complex, a type of group complex, was invented by A.L. Blakers under the name of group system; see $[\mathbf{3}]$. In $[\mathbf{3 8}, \mathbf{3 9}]$, J.H.C. Whitehead re-introduced them, considering, however, only the important totally free case. Crossed complexes algebraically describe, for instance, the complex of groups

$$
\cdots \stackrel{\partial_{5}}{\longrightarrow} \pi_{4}\left(M^{4}, M^{3}, *\right) \stackrel{\partial_{4}}{\longrightarrow} \pi_{3}\left(M^{3}, M^{2}, *\right) \stackrel{\partial_{3}}{\longrightarrow} \pi_{2}\left(M^{2}, M^{1}, *\right) \stackrel{\partial_{2}}{\longrightarrow} \pi_{1}\left(M^{1}, *\right),
$$

with the obvious boundary maps $\partial_{n}, n \in \mathbb{N}$, together with the standard action of $\pi_{1}\left(M^{1}, *\right)$ on $\pi_{n}\left(M^{n}, M^{n-1}, *\right), n>1$. Here $M$ is a connected CW-complex and $M^{n}$ is its $n$-skeleton. This crossed complex, denoted by $\Pi(M)$, is called the fundamental crossed complex of $M$ (provided with its skeletal filtration). More generally, we can take $M$ to be any filtered space.

What distinguishes a crossed complex from a chain complex of groups, is that a crossed complex is also provided with an action of the first group on all the others, satisfying some natural compatibility conditions, which are stronger than merely requiring that the boundary maps preserve the actions.

Crossed complexes were extensively studied and used, for example, by J.H.C. Whitehead, H.J. Baues, R. Brown and P.J. Higgins; see $[\mathbf{3 8}, \mathbf{3 9}, \mathbf{1 ,}$, , 5, 10, 11, 12, 13, 14]. They admit an obvious notion of homotopy, as well as classifying spaces. Another important feature is that the category of crossed complexes is a category with colimits. Thus, in particular, the free product of any family of crossed complexes is well defined. In addition, there exists a general "van Kampen type property", stating that, under mild conditions, the crossed complex functor from the category of filtered spaces to the category of crossed complexes preserves colimits. This strong result is due to R. Brown and P.J. Higgins, and appeared in $[\mathbf{1 1}, \mathbf{1 2}]$. We will refer to it as the "Higher Homotopy van Kampen Theorem". Another usual designation is "Generalised van Kampen Theorem". I In particular, the fundamental crossed complex functor from the category of CW-complexes to the category of crossed complexes preserves colimits.

Let $M$ be any space which can be given the structure of a $\mathrm{CW}$-complex. The fundamental crossed complex $\Pi(M)$ of the skeletal filtration of $M$ is strongly dependent on the chosen cellular structure. However, J.H.C. Whitehead proved (see [38]) that if $M$ is a CW-complex, then the homotopy type of the crossed complex $\Pi(M)$ depends only on the homotopy type of $M$ as a space. In fact, the results of J.H.C. Whitehead that appeared in $[\mathbf{3 8}, 39]$ immediately imply a stronger result: If $M$ and $N$ are homotopic CW-complexes of dimension $\leqslant n$, then $\Pi\left(M^{\prime}\right)$ and $\Pi\left(N^{\prime}\right)$ are simply homotopy equivalent. Here $M^{\prime}$ and $N^{\prime}$ are obtained from $M$ and $N$ by

\footnotetext{
${ }^{1} \mathrm{Jim}$ Stasheff suggested recently to Ronnie Brown that the designation "Higher Homotopy van Kampen Theorem" would be more informative than the previous "Generalised van Kampen Theorem".
} 
taking wedge products with a certain number of spheres $S^{n}$. We refer to [39] for the definition of simple homotopy equivalence of crossed complexes.

In this article we prove a theorem (see Theorem 4.1) expanding the first of the results due to J.H.C. Whitehead which we referred to in the previous paragraph; a result in the direction of the second of them. That is, we show that $\Pi(M)$ depends only on the homotopy type of $M$, as a space, up to free products with crossed complexes of the type $\mathcal{D}^{n} \doteq \Pi\left(D^{n}\right)$, where $n \in \mathbb{N}$. Here $D^{n}$ is the $n$-disk with its natural CW-decomposition with one 0-cell, one $(n-1)$-cell and one $n$-cell. This theorem is deduced (making use of the Higher Homotopy van Kampen Theorem) from an analogous statement on CW-complexes (proved in this article): The homotopy type of a CW-complex $M$, as a filtered space (in other words of the skeletal filtration of $M$ ), depends only on the homotopy type of $M$ (as a space), up to wedge products with CW-complexes of the type $D^{n}$ (where $n \in \mathbb{N}$ ) provided with their natural cell decompositions, already described. These results (probably the strongest in this article) are contained in Theorems 3.3, 3.4 and 3.5. See also Subsection 3.3 for the less complicated though very suggestive crossed module case. Note that Theorem 4.1 corresponds algebraically to Theorem 3.4, and this follows directly from the Higher Homotopy van Kampen Theorem.

These types of statements have strong similarities with the following result of J.H.C. Whitehead on simple homotopy types of CW-complexes: If $M$ and $N$ are $\mathrm{CW}$-complexes of dimension $\leqslant n$, and $f: M \rightarrow N$ is a $(n-1)$-equivalence, then $M^{\prime}$ and $N^{\prime}$ are simply homotopy equivalent, where $M^{\prime}$ and $N^{\prime}$ are obtained from $M$ and $N$ by taking wedge products with a certain number of spheres $S^{n}$. Note that the previously stated result on the simple homotopy types of $\Pi(M)$ and $\Pi(N)$ can be deduced from this statement together with the Higher Homotopy van Kampen Theorem.

As an application, we prove that if $\mathcal{A}$ is a finite crossed complex, then the number of morphisms from $\Pi(M)$ into $\mathcal{A}$ can be naturally normalised to a homotopy invariant $I_{\mathcal{A}}(M)$ of $\mathrm{CW}$-complexes $M$, which is as easy to calculate as the cellular homology groups of $M$. This invariant generalises a previous construction for crossed modules appearing in [20]. There it was proved that, if $\mathcal{A}$ is a crossed module, then the value of $I_{\mathcal{A}}$ on a knot complement defines a non-trivial invariant of knotted surfaces $\Sigma$ embedded in $S^{4}$. Moreover, we elucidated a graphical algorithm for its calculation from a movie presentation of $\Sigma$.

The homotopy invariant $I_{\mathcal{A}}$ also upgrades previous constructions of invariants of manifolds and knots derived from finite crossed modules; see $[40,30, \mathbf{1 9}]$. Another construction by T. Porter appearing in [31] (from which TQFTs can be defined) considers finite $n$-cat groups, which are more general than finite crossed complexes. Finally, in [25], M. Mackaay defined 4-manifold invariants, conjecturally related to the $n=3$ case of T. Porter's construction, but considering, furthermore, an additional twisting by cohomology classes of 3-types. This twisting is similar to the one R. Dijkgraaf and E. Witten introduced in [16], even though their 3-dimensional oriented manifold invariant (the well known Dijkgraaf-Witten invariant) considers only finite 1-types (in other words, finite groups).

At the end of this article, we interpret and give an alternative proof of the 
existence of the invariant $I_{\mathcal{A}}$ where $\mathcal{A}$ is a finite crossed complex. Namely, we prove that $I_{\mathcal{A}}(M)$ is determined by the number of connected components as well as the homotopy groups of the function space $\operatorname{TOP}((M, *),(|\mathcal{A}|, *))$, where $|\mathcal{A}|$ is the classifying space of $\mathcal{A}$, using a multiplicative Euler characteristic-type formula. This result is an application of the general theory of classifying spaces of crossed complexes developed by R. Brown and P.J. Higgins (see [13]), and also of the non-trivial fact that given two crossed complexes $\mathcal{A}$ and $\mathcal{B}$ we can define a crossed complex $C R S(\mathcal{A}, \mathcal{B})$, made from all crossed complex morphisms $\mathcal{A} \rightarrow \mathcal{B}$ together with their $n$-fold homotopies, where $n \in \mathbb{N}$. In fact, given a CW-complex $M$ with a unique 0-cell, there exists a weak homotopy equivalence $|C R S(\Pi(M), \mathcal{A})| \rightarrow$ $\operatorname{TOP}((M, *),(|\mathcal{A}, *|))$; see $[\mathbf{1 1}, \mathbf{1 2}, \mathbf{1 3}]$. Therefore, the weak homotopy type of the function space $\operatorname{TOP}((M, *),(|\mathcal{A}, *|))$ can be fully described in algebraic terms.

This description of $I_{\mathcal{A}}$ enables us to incorporate $n$-dimensional cohomology classes of $|\mathcal{A}|$ into it, as long as we consider only $n$-dimensional closed oriented manifolds. This therefore yields an extension of Dijkgraaf-Witten's invariant to crossed complexes. Its full description will appear in a future joint paper; see [21].

\section{Acknowledgements}

This work was funded by Fundação para a Ciência e a Tecnologia (Portugal), post-doctoral grant SFRH/BPD/17552/2004, part of the research project POCI/ MAT/60352/2004 ("Quantum Topology"), also financed by FCT and cofinanced by the European Community fund FEDER.

I would like to express my gratitude to Gustavo Granja for many helpful discussions, which were extremely important for the development of this work. I also want to thank Tim Porter and Ronnie Brown for introducing me to this subject, and for a large amount of further suggestions and help, which influenced this article, and finally Roger Picken for his constant support.

\section{Preliminaries}

All CW-complexes considered in this article will be connected, with an extra technical condition imposed, namely that they have a unique 0 -cell, taken to be their base point. The only exception is Subsection 2.1. We will often make the assumption that they have only a finite number of $n$-cells for each $n \in \mathbb{N}$. If $M$ is a CW-complex, denote the $n$-skeleton of $M$ by $M^{n}$, where $n \in \mathbb{N}$. If $f: M \rightarrow N$ is a cellular map, where $N$ is a CW-complex, denote $f^{n} \doteq f_{\mid M^{n}}: M^{n} \rightarrow N^{n}$. If $n$ is an integer, then an $n$-type is a CW-complex $M$ such that $\pi_{k}(M)=\{0\}$ if $k>n$. Set $I=[0,1]$.

\subsection{Cofibred filtrations}

Most issues treated in this subsection are widely known. The specialist should pass directly to Section 3, and use this subsection, as well as the next one on crossed complexes, only as a reference. 
Definition 2.1. Let $M$ be some (Hausdorff) space. A filtration of $M$ is a sequence $\left\{M_{n}\right\}_{n=0}^{\infty}$ (briefly $\left\{M_{n}\right\}$ ) of subspaces of $M$ such that $M_{n} \subset M_{n+1}, \forall n \in \mathbb{N}$, and also $M=\bigcup_{n \in \mathbb{N}} M_{n}$. A space $M$ provided with a filtration is called a filtered space. A filtration of $M$ is called finite, of length $L \in \mathbb{N}$, if $M_{n}=M, \forall n \geqslant L$. A filtration $\left\{M_{n}\right\}_{n \in \mathbb{N}}$ of $M$ is called cofibred if the inclusion $M_{n} \rightarrow M_{n+1}$ is a cofibration for each $n \in \mathbb{N}$. If $M$ and $N$ are filtered topological spaces, then a filtered map $f: M \rightarrow N$ is a continuous map such that $f\left(M_{n}\right) \subset N_{n}, \forall n \in \mathbb{N}$. A filtered homotopy between the filtered maps $f, g: M \rightarrow N$ is a homotopy $H: M \times I \rightarrow N$ such that $H\left(M_{n} \times I\right) \subset$ $N_{n}$ for each $n \in \mathbb{N}$. Filtered homotopy is an equivalence relation.

Obviously filtered topological spaces and filtered maps form a category. If $f$ : $M \rightarrow N$ is a filtered map, we set $f_{n} \doteq f_{\mid M_{n}}: M_{n} \rightarrow N_{n}$, where $n \in \mathbb{N}$. There exists a category whose objects are filtered topological spaces, with morphisms being the filtered homotopy classes of filtered maps.

Example 2.2. Let $M$ be a CW-complex. Then $M$ has a natural cofibred filtration $\left\{M^{n}\right\}_{n \in \mathbb{N}}$, where $M^{n}$ is the n-skeleton of $M$, the skeletal filtration of $M$. More generally, if $\left\{M_{n}\right\}_{n \in \mathbb{N}}$ is a filtration of $M$, such that each $M_{n}$ is a CW-complex included in $M_{n+1}$, cellularly, for any $n \in \mathbb{N}$, then $\left\{M_{n}\right\}_{n \in \mathbb{N}}$ is a cofibred filtration. In fact, all the filtrations that we consider in this article will be of this particular type, called filtrations by subcomplexes.

2.1.1. Some results on cofibre homotopy equivalence The following result is well known:

Theorem 2.3. Let $M$ and $N$ be spaces provided with some cofibred filtrations, say $\left\{M_{n}\right\}_{n \in \mathbb{N}}$ and $\left\{N_{n}\right\}_{n \in \mathbb{N}}$, which we suppose to be finite (we will eliminate this condition later, in the cellular case). Let $F: M \rightarrow N$ be a filtered map. Suppose that each map $F_{n}: M_{n} \rightarrow N_{n}, n \in \mathbb{N}$ is a homotopy equivalence. Then it follows that $F$ is a filtered homotopy equivalence.

This is shown in $[\mathbf{2 6}$, end of chapter 6], and in $[\mathbf{6}, 7.4]$ for the case in which both filtrations of $M$ and $N$ have length $L=1$. The proof given in [6] extends immediately to the general case, inductively, as we will indicate below. Later we will give an alternative proof of Theorem 2.3 for the particular case of filtrations by subcomplexes, which adapts to infinite filtrations, a generality we will need in order to be able to work with infinite dimensional $\mathrm{CW}$-complexes.

It is convenient to recall the following result appearing in $[\mathbf{6}, 7.4]$, which leads immediately to a proof of Theorem 2.3.

Lemma 2.4 (R. Brown). Let $F:\left(M_{1}, M_{0}\right) \rightarrow\left(N_{1}, N_{0}\right)$ be a map between cofibred pairs. Suppose that $F=F_{1}: M_{1} \rightarrow N_{1}$ and $F_{0}: M_{0} \rightarrow N_{0}$ are homotopy equivalences. Let $G_{0}: N_{0} \rightarrow M_{0}$ be a homotopy inverse of $F_{0}$, and consider homotopies (in $M_{0}$ and $\left.N_{0}\right)$;

$$
K: G_{0} F_{0} \rightarrow \operatorname{id}_{M_{0}} \text { and } H: F_{0} G_{0} \rightarrow \operatorname{id}_{N_{0}} .
$$

Then $G_{0}$ extends to a homotopy inverse $G$ of $F$ such that there exists not only a homotopy $F G \rightarrow \mathrm{id}_{N_{1}}$ which extends $H$, but also a homotopy $G F \rightarrow \mathrm{id}_{M_{1}}$ extending 
the concatenation $J$ of the following homotopies:

$$
G_{0} F_{0} \stackrel{G_{0} F_{0} K^{-1}}{\longrightarrow} G_{0} F_{0} G_{0} F_{0} \stackrel{G_{0} H F_{0}}{\longrightarrow} G_{0} F_{0} \stackrel{K}{\longrightarrow} \operatorname{id}_{M_{0}} .
$$

Note that if $G_{0}, F_{0}, H, K$ are filtered, for some filtrations of $M_{0}$ and $N_{0}$, then so is the homotopy $J: G_{0} F_{0} \rightarrow \operatorname{id}_{M_{0}}$.

Theorem 2.3 can therefore be strengthened in the following way.

Corollary 2.5. Under the conditions of Theorem 2.3, if

$$
G_{L}:\left(N_{L}, N_{L-1}, \ldots, N_{0}\right) \rightarrow\left(M_{L}, M_{L-1}, \ldots, M_{0}\right)
$$

is a filtered homotopy inverse of the filtered map

$$
F_{L}=F_{\mid M_{L}}:\left(M_{L}, M_{L-1}, \ldots, M_{0}\right) \rightarrow\left(N_{L}, N_{L-1}, \ldots, N_{0}\right),
$$

then there exists a filtered homotopy inverse $G$ of $F$ extending $G_{L}$.

The following is the main lemma of this subsection, crucial for the proof of the main theorem of this article, Theorem 3.3:

Lemma 2.6. Let $\left\{M_{n}\right\}_{n \in \mathbb{N}}$ and $\left\{N_{n}\right\}_{n \in \mathbb{N}}$ be filtrations of the $C W$-complexes $M$ and $N$, so that each $M_{n}$ (respectively $N_{n}$ ) is also a $C W$-complex, included in $M$ (respectively $N)$, cellularly, for each $n \in \mathbb{N}$. Suppose that $M$ is embedded in $N$, cellularly, and that ${ }^{2} M_{n}=M \cap N_{n}, \forall n \in \mathbb{N}$. If, for each $n \in \mathbb{N}$, the inclusion of $M_{n}$ in $N_{n}$ is a homotopy equivalence, then there exists a deformation retraction from $N$ onto $M$, say $\rho: N \times I \rightarrow N$, such that the restriction of $\rho$ to $N_{n} \times I$ is a deformation retraction of $N_{n}$ in $M_{n}$, for each $n \in \mathbb{N}$. In fact $\rho$ can be chosen to be cellular, in the sense that the map $\rho: N \times I \rightarrow N$ is cellular.

Definition 2.7. Deformation retractions with this property will be called filtered.

Proof of Lemma 2.6. Suppose first that the filtrations of $M$ and $N$ have length $L=1$. There exists a deformation retraction $\rho_{0}: N_{0} \times I \rightarrow N_{0}$, of $N_{0}$ in $M_{0}$, which we can suppose to be cellular. Let $r: N_{1} \times I \rightarrow N_{1}$ be a deformation retraction of $N_{1}$ in $M_{1}$, also chosen to be cellular. Consider the CW-complex $N_{1} \times I$. Let us define a function $\rho_{1}: N_{1} \times I \rightarrow N_{1}$, in such a way that $\rho_{1}$ is a cellular deformation retraction from $N_{1}$ onto $M_{1}$, extending $\rho_{0}$ (thus permitting the argument to be extended to the general case by induction). We define $\rho_{1}\left(m_{1}, t\right)=m_{1}, \forall m_{1} \in M_{1}, \forall t \in I$. Define also $\rho_{1}\left(n_{0}, t\right)=\rho_{0}\left(n_{0}, t\right), \forall n_{0} \in N_{0}, \forall t \in I$, and $\rho_{1}\left(n_{1}, 0\right)=n_{1}, \forall n_{1} \in N_{1}$. There is no contradiction since $M_{1} \cap N_{0}=M_{0}$, and $\rho_{0}$ is a deformation retraction of $N_{0}$ in $M_{0}$.

Let us now extend $\rho_{1}$ to all of $N_{1} \times I$. Let $k \in \mathbb{N}$. Suppose we have already extended the function $\rho_{1}$ to $\left(N_{1}^{k-1} \times I\right) \cup\left(N_{0} \times I\right) \cup\left(M_{1} \times I\right) \cup\left(N_{1} \times\{0\}\right)$, yielding a cellular map, with $\rho_{1}\left(N_{1}^{k-1} \times\{1\} \cup N_{0} \times\{1\} \cup M_{1} \times\{1\}\right) \subset M_{1}$. Let $e^{k}$ be a $k$ cell of $N_{1}$ not belonging to $M_{1} \cup N_{0}$. The function $\rho_{1}$ is already defined in $\partial e^{k} \times I$ and $e_{k} \times\{0\}$. Moreover $\rho_{1}\left(\partial e^{k} \times\{1\}\right) \subset M_{1}$. We can extend $\rho_{1}$ to all $e_{k} \times I$ by

${ }^{2}$ Recall that if $A$ and $B$ are subcomplexes of a CW-complex $C$, then $A \cap B$ is a subcomplex of $C$ with a cell decomposition consisting of the cells of $C$ occurring in both $A$ and $B$. 
using the cellular deformation retraction $r: N_{1} \times I \rightarrow N_{1}$ (from $N_{1}$ to $M_{1}$ ). Let us be explicit. Choose a homeomorphism

$$
f: \overline{e^{k}} \times I \rightarrow\left(\partial e^{k} \times I \cup e^{k} \times\{0\}\right) \times I
$$

such that $f\left(\overline{e^{k}} \times\{1\}\right) \subset\left(\partial e^{k} \times I \cup e^{k} \times\{0\}\right) \times\{1\} \cup\left(\partial e^{k} \times\{1\}\right) \times I$, and $f$ sends $\left(\partial e^{k} \times I \cup e^{k} \times\{0\}\right) \subset \overline{e^{k}} \times I$ identically to its copy $\left(\partial e^{k} \times I \cup e^{k} \times\{0\}\right) \times\{0\} \subset$ $\left(\partial e^{k} \times I \cup e^{k} \times\{0\}\right) \times I$. In particular, $f$ is cellular. Then we extend $\rho_{1}$ to $e^{k} \times I$ in the following way:

$$
\rho_{1}(x, s)=r\left(\left(\rho_{1} \times \mathrm{id}\right) \circ f(x, s)\right), x \in e^{k}, s \in I .
$$

Therefore $\rho_{1}\left(e^{k} \times\{1\}\right) \subset M_{1}$, and the function obtained is still cellular. The result for $L=2$ follows from induction in $k$. As we have seen, the general case follows from the proof of the case $L=1$ by an inductive argument.

Let $M$ and $N$ be CW-complexes. Suppose that they have unique 0 -cells, which we take to be their base points $*$. Give both $M$ and $N$ filtrations $\left\{M_{n}\right\}$ and $\left\{N_{n}\right\}$ by subcomplexes. Let $f:(M, *) \rightarrow(N, *)$ be a filtered map, which we suppose, furthermore, to be cellular. Consider the mapping cylinder

$$
\mathcal{M}_{f}=M \times I \bigcup_{(x, 1) \mapsto f(x)} N
$$

of $f$. Recall that $\mathcal{M}_{f}$ has a natural deformation retraction onto $N$, say $\rho: \mathcal{M}_{f} \times I \rightarrow$ $\mathcal{M}_{f}$, obtained by sliding the segment $\{x\} \times I$ (where $x \in M$ ) along itself, towards the endpoint $f(x) \in N$. Therefore there exists a natural retraction $r: \mathcal{M}_{f} \rightarrow N$, where $r(x)=\rho(x, 1), \forall x \in \mathcal{M}_{f}$; in other words $r(a, t)=f(a) \forall a \in M, \forall t \in I$ and $r(b)=b$, $\forall b \in N$. Note that $f: M \rightarrow N$ is $r$ composed with the inclusion map $i: M \rightarrow \mathcal{M}_{f}$.

The mapping cylinder $\mathcal{M}_{f}$ of $f: M \rightarrow N$ has a natural cell decomposition, with $M$ and $N$ contained in $\mathcal{M}_{f}$, cellularly. Additionally, we have an $(n+1)$-cell of $\mathcal{M}_{f}$, for each $n$-cell $e^{n}$ of $M$, connecting $e^{n}$ with $f\left(e^{n}\right)$. This makes sense since $f$ is cellular.

The reduced mapping cylinder $\mathcal{M}_{f}^{\prime}$ of $f$ is obtained from the CW-complex $\mathcal{M}_{f}$ by collapsing the 1-cell of $\mathcal{M}_{f}$ connecting $* \in M$ with $* \in N$ to a point. Therefore $\mathcal{M}_{f}^{\prime}$ has a unique 0 -cell (which we also call $*$ ), and both $M$ and $N$ are included in $\mathcal{M}_{f}^{\prime}$, cellularly, although intersecting in $* \in \mathcal{M}_{f}^{\prime}$. If $f$ is a homotopy equivalence, then $M$ is a deformation retract of $\mathcal{M}_{f}^{\prime}$.

The CW-complex $\mathcal{M}_{f}^{\prime}$ is filtered, in a natural way, by the reduced mapping cylinders $\mathcal{M}_{f_{n}}^{\prime}$ of the restrictions $f_{n}: M_{n} \rightarrow N_{n}$ of $f$ to $M_{n}$, where $n \in \mathbb{N}$. Note that $\mathcal{M}_{f_{n}}^{\prime}$ is included in $\mathcal{M}_{f}^{\prime}$, cellularly, for any $n \in \mathbb{N}$. In addition, both $M_{n}$ and $N_{n}$ are embedded in $\mathcal{M}_{f_{n}}^{\prime}$, cellularly, and, moreover, $M_{n}=\mathcal{M}_{f_{n}}^{\prime} \cap M$ and $N_{n}=\mathcal{M}_{f_{n}}^{\prime} \cap N$, for any $n \in \mathbb{N}$. From Lemma 2.6 it follows that:

Corollary 2.8. Let $M$ and $N$ be $C W$-complexes with a unique 0 -cell, provided with filtrations $\left\{M_{n}\right\}$ and $\left\{N_{n}\right\}$ by subcomplexes. Let $f: M \rightarrow N$ be a filtered map, which we assume to be cellular, such that the restriction $f_{n}$ of $f$ to $M_{n}$ is a homotopy equivalence $f_{n}: M_{n} \rightarrow N_{n}$, for each $n \in \mathbb{N}$. There exist filtered deformation retractions of $\mathcal{M}_{f}^{\prime}$ onto $M$ (respectively of $\mathcal{M}_{f}^{\prime}$ onto $N$ ), say $\rho, \rho^{\prime}: \mathcal{M}_{f}^{\prime} \times I \rightarrow \mathcal{M}_{f}^{\prime}$ (respectively). 
In other words, the restrictions of $\rho$ and $\rho^{\prime}$ to $\mathcal{M}_{f_{n}}^{\prime} \times I$ are deformation retractions from $\mathcal{M}_{f_{n}}^{\prime}$ to $M_{n}$ (respectively from $\mathcal{M}_{f_{n}}^{\prime}$ to $N_{n}$ ), for any $n \in \mathbb{N}$.

Irrespectively of $f: M \rightarrow N$ being a homotopy equivalence or not, the reduced mapping cylinder $\mathcal{M}_{f}^{\prime}$ of $f$ always deformation retracts onto $N$, in the same way that the mapping cylinder $\mathcal{M}_{f}$ of $f$ does. This deformation retraction $\rho: \mathcal{M}_{f}^{\prime} \times I \rightarrow \mathcal{M}_{f}^{\prime}$, induced by the natural deformation retraction of $\mathcal{M}_{f}$ onto $N$, is filtered. Therefore the obvious retraction $R: \mathcal{M}_{f}^{\prime} \rightarrow N$, such that $R(x)=\rho(x, 1), \forall x \in \mathcal{M}_{f}^{\prime}$ (thus $R(a, t)=f(a), \forall a \in M, \forall t \in I$ and $R(b)=b, \forall b \in N)$, is a filtered homotopy inverse of the inclusion map $j: N \rightarrow \mathcal{M}_{f}^{\prime}$. As before, we have $f=R \circ i$, where $i: M \rightarrow \mathcal{M}_{f}$ is the inclusion map. These very simple facts will be essential later.

Note that Corollary 2.8 is valid without the assumption that the CW-complexes $M$ and $N$ have unique 0 -cells, considering mapping cylinders instead of reduced mapping cylinders. As a consequence, we obtain an extension of Theorem 2.3 for infinite filtrations, as long as they are filtrations by subcomplexes of $\mathrm{CW}$-complexes and all maps considered are cellular.

Corollary 2.9. Let $M$ and $N$ be $C W$-complexes equipped with filtrations $\left\{M_{n}\right\}$ and $\left\{N_{n}\right\}$ by subcomplexes of $M$ and $N$ respectively. Let $f: M \rightarrow N$ be a filtered map, which we suppose, furthermore, to be cellular. Suppose that $f_{n}: M_{n} \rightarrow N_{n}$ is a homotopy equivalence for each $n \in \mathbb{N}$. Then $f$ is a filtered homotopy equivalence.

Proof. Consider the filtration of the mapping cylinder $\mathcal{M}_{f}$ of $f$ by the mapping cylinders $\mathcal{M}_{f_{n}}$ of the restrictions $f_{n}: M_{n} \rightarrow N_{n}$ of $f$ to $M_{n}$, where $n \in \mathbb{N}$. Then, by the previous corollary, there exist filtered homotopy equivalences $M \cong \mathcal{M}_{f} \cong$ $N$. Specifically, the inclusions $i: M \rightarrow \mathcal{M}_{f}$ and $j: N \rightarrow \mathcal{M}_{f}$ are filtered homotopy equivalences. The obvious retraction $r: \mathcal{M}_{f} \rightarrow N$ is a filtered homotopy inverse of $j: N \rightarrow \mathcal{M}_{f}$. The result follows from the fact that $r \circ i=f$.

\subsection{Crossed complexes}

This subsection will only be needed in Section 3. We gather some results on crossed complexes which we will use. Nothing here is new, and most is due to R. Brown and P.J. Higgins. The exceptions are Subsections 2.2.1 and 2.2.4, whose results are mainly due to J.H.C. Whitehead.

Let $G$ and $E$ be groups. Recall that a crossed module with base $G$ and fibre $E$, say $\mathcal{G}=(G, E, \partial, \triangleright)$, is given by a group morphism $\partial: E \rightarrow G$ and an action $\triangleright$ of $G$ on $E$ on the left by automorphisms. The conditions on $\triangleright$ and $\partial$ are

1. $\partial(X \triangleright e)=X \partial(e) X^{-1}, \forall X \in G, \forall e \in E$,

2. $\partial(e) \triangleright f=e f e^{-1}, \forall e, f \in E$.

Notice that the second condition implies that ker $\partial$ commutes with all of $E$. We call $G$ the base group and $E$ the principal group. A morphism $F=(\phi, \psi)$ between the crossed modules $\mathcal{G}$ and $\mathcal{G}^{\prime}=\left(G^{\prime}, E^{\prime}, \partial^{\prime}, \triangleright^{\prime}\right)$ is given by a pair of group morphisms 
$\phi: G \rightarrow G^{\prime}$ and $\psi: E \rightarrow E^{\prime}$, making the diagram

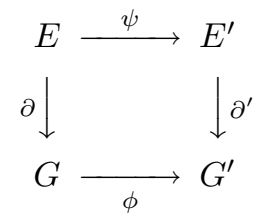

commutative. In addition we must have:

$$
\phi(X) \triangleright^{\prime} \psi(e)=\psi(X \triangleright e), \forall X \in G, \forall e \in E .
$$

There exists an extensive literature on crossed modules. We refer, for example to $[\mathbf{1}, \mathbf{2}, \mathbf{5}, \mathbf{8}, \mathbf{9}, \mathbf{1 5}, \mathbf{1 4}, \mathbf{2 0}]$. A natural generalisation of the concept of a crossed module is a crossed complex:

Definition 2.10. A (reduced) crossed complex $\mathcal{A}$ is given by a complex of groups

$$
\cdots \rightarrow A_{n} \stackrel{\partial_{n}=\partial}{\longrightarrow} A_{n-1} \stackrel{\partial_{n-1}=\partial}{\longrightarrow} A_{n-2} \rightarrow \cdots \rightarrow A_{2} \stackrel{\partial_{2}=\partial}{\longrightarrow} A_{1}(\stackrel{p}{\rightarrow} A \rightarrow\{1\})
$$

such that:

1. There exists a left action $\triangleright=\triangleright_{n}$ of the group $A_{1}$ on $A_{n}$, by automorphisms, for any $n \in \mathbb{N}$, and all the boundary maps $\partial$ are $A_{1}$-module morphisms. In addition, we suppose that $A_{1}$ acts on itself by conjugation.

2. The map $A_{2} \stackrel{\partial_{2}}{\longrightarrow} A_{1}$ together with the action $\triangleright$ of $A_{1}$ in $A_{2}$ defines a crossed module. In other words, to (1) we add the condition $\partial(e) \triangleright f=e f e^{-1}, \forall e, f \in$ $A_{2}$.

3. The group $A_{n}$ is abelian if $n>2$.

4. The action of $A_{1}$ on $A_{n}$ factors through the projection $p: A_{1} \rightarrow A=\operatorname{coker}\left(\partial_{2}\right)$, for $n>2$, and, in particular, $A$ acts on $A_{n}, n>2$, on the left, by automorphisms.

If $L \geqslant 1$ is integer, an $L$-truncated crossed complex is a crossed complex such that $A_{n}=\{0\}$ if $n>L$.

A natural example of a crossed complex is the following one, introduced by A.L. Blakers in [3]:

Example 2.11. Let $M$ be a path-connected space, and let $\left\{M_{n}\right\}, n \in \mathbb{N}$ be a filtration of it, where all spaces $M_{n}, n \in \mathbb{N}$ are path-connected and $M_{0}$ is a singleton, which we take to be the base point $*$. Then the sequence of groups $\pi_{n}\left(M_{n}, M_{n-1}, *\right)$, where $n=2,3, \ldots$, together with $\pi_{1}\left(M_{1}, *\right)$, with the obvious boundary maps and left actions of $\pi_{1}\left(M_{1}, *\right)$ on them is a crossed complex, which we denote by $\Pi(M)$ and call the fundamental crossed complex of the filtered space $M$. If the filtration of $M$ is finite, of length $L \in \mathbb{N}$, then $\Pi(M)$ is an $L$-truncated crossed complex, which we denote by $\Pi_{L}\left(M, M_{L-1}, \ldots, M_{2}, M_{1}, *\right)$.

If $M$ and $N$ are filtered spaces and $f: M \rightarrow N$ is a filtered map, we denote the induced map on crossed complexes by $f_{*}: \Pi(M) \rightarrow \Pi(N)$. Note that if $f$ and 
$g$ are filtered homotopic, then the induced maps on crossed complexes coincide. However this is not the case when $f$ and $g$ are merely homotopic as maps (but see Theorem 2.26).

Remark 2.12. Let $M$ be a CW-complex with a unique 0 -cell, which we take to be its base point. The notation $\Pi(M)$ will always mean the fundamental crossed complex of the skeletal filtration of $M$. If $L \in \mathbb{N}$, we will also denote $\Pi_{L}(M) \doteq$ $\Pi_{L}\left(M, M^{L-1}, \ldots, M^{1}, M^{0}=*\right)$. This type of crossed complex was considered by J.H.C. Whitehead in $[\mathbf{3 8}, \mathbf{3 9}]$.

It is easy to show that crossed complexes and their morphisms, defined in the obvious way, form a category. Crossed complexes are studied or used extensively in $[1,2,5,8,10,11,12,13,14,38,39]$, for example. Notice that H.J. Baues calls them "crossed chain complexes". J.H.C. Whitehead considered only totally free crossed complexes, to be defined below, referring to them as "homotopy systems".

We will usually denote a crossed complex $\mathcal{A}$ by $\mathcal{A}=\left(A_{n}, \partial_{n}, \triangleright_{n}\right)$, or more simply by $\left(A_{n}, \partial_{n}\right)$, or even $\left(A_{n}\right)$. A morphism $f: \mathcal{A} \rightarrow \mathcal{B}$ of crossed complexes will normally be denoted by $f=\left(f_{n}\right)$.

The category of crossed complexes is a category with colimits. See $[\mathbf{1 0}, \mathbf{1 1}, \mathbf{1 2}]$. In particular, we can consider the free product $\mathcal{A} \vee \mathcal{B}$ of two crossed complexes, defined as the pushout of the diagram

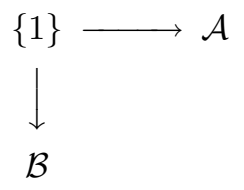

and analogously for free products of infinite families of crossed complexes. Here $\{1\}$ is the trivial crossed complex, so that $\{1\}_{n}=\{1\}, \forall n \in \mathbb{N}$. Therefore, if $\mathcal{C}$ is a crossed complex, there exists a one-to-one correspondence between $\operatorname{Hom}(\mathcal{A} \vee \mathcal{B}, \mathcal{C})$ and $\operatorname{Hom}(\mathcal{A}, \mathcal{C}) \times \operatorname{Hom}(\mathcal{B}, \mathcal{C})$.

We will not need the explicit construction of $\mathcal{A} \vee \mathcal{B}$, although we will use the concept of free product extensively.

Remark 2.13. The definition of crossed complexes we gave is a particular case of a more general definition. Indeed, we can consider that $A_{1}$ is a groupoid with set of objects $C$, and that each $A_{n}$ is a totally disconnected groupoid over the same set $C$. In addition, all group actions must be substituted by groupoid actions, in the obvious way. See $[\mathbf{5}, \mathbf{7}, \mathbf{1 0}, \mathbf{1 1}]$, for example, for a discussion of crossed complexes in the groupoid context. This full generality will be needed later for the definition of $C R S(\mathcal{A}, \mathcal{B})$ in 2.2.5. We will use the designations "reduced" and "non-reduced", to distinguish between the group and groupoid-based definitions of crossed complexes, respectively, whenever ambiguity may arise.

It is important to note that the inclusion of the category of reduced crossed complexes into the category of (non-reduced) crossed complexes preserves colimits of connected diagrams, thus, in particular, pushouts. 


\subsubsection{Totally free crossed complexes}

Definition 2.14. Consider a group $G$. Let $K$ be a set and $\partial_{0}: K \rightarrow G$ be a map. A crossed module $\mathcal{G}=(G, E, \partial, \triangleright)$ is said to be the free crossed module on $K$ and $\partial_{0}: K \rightarrow G$ if there exists an injective map $i: K \rightarrow E$ such that $\partial \circ i=\partial_{0}$, and the following universal property is satisfied:

For any crossed module $\mathcal{G}^{\prime}=\left(G^{\prime}, E^{\prime}, \partial^{\prime}, \triangleright^{\prime}\right)$, any group morphism $\phi: G \rightarrow G^{\prime}$, and any map $\psi_{0}: K \rightarrow E^{\prime}$ for which $\partial^{\prime} \circ \psi_{0}=\phi \circ \partial_{0}$, there exists a unique crossed module map $(\phi, \psi): \mathcal{G} \rightarrow \mathcal{G}^{\prime}$ such that $\psi \circ i=\psi_{0}$.

For more details on the construction of these free crossed modules (defined up to isomorphism), we refer the reader, for example, to $[\mathbf{1 4}, \mathbf{8 ,} \mathbf{1 5}, \mathbf{2 0}$ ].

Recall the following theorem, due to J.H.C. Whitehead. For the original proof, see $[\mathbf{3 5}, \mathbf{3 6}, \mathbf{3 8}]$. See also $[\mathbf{1 4}, 5.4]$ and $[\mathbf{7}, \mathbf{1 0}, \mathbf{2 2}]$.

Theorem 2.15 (Whitehead's Theorem). Let $M$ be a path-connected space with a base point $*$. Let $N$ be a space obtained from $M$ by attaching some 2-cells $s_{1}, \ldots$, $s_{n}$. Each 2-cell $s_{i}$ therefore induces an element $\phi\left(s_{i}\right)$ of $\pi_{1}(M, *)$ (defined up to conjugation by an element of $\left.\pi_{1}(M, *)\right)$ through its attaching map. Then the crossed module $\Pi_{2}(N, M, *)$ is the free crossed module over the map $\phi:\left\{s_{1}, \ldots, s_{n}\right\} \rightarrow$ $\pi_{1}(M, *)$.

Definition 2.16. A (reduced) crossed complex $\mathcal{A}$ of the form

$$
\cdots \rightarrow A_{n} \stackrel{\partial_{n}=\partial}{\longrightarrow} A_{n-1} \stackrel{\partial_{n-1}=\partial}{\longrightarrow} A_{n-2} \rightarrow \cdots \rightarrow A_{2} \stackrel{\partial_{2}=\partial}{\longrightarrow} A_{1} \stackrel{p}{\rightarrow} A \rightarrow\{1\}
$$

is said to be totally free if:

1. $A_{1}$ is the free group on a set $C_{1}$.

2. The map $A_{2} \stackrel{\partial_{2}}{\longrightarrow} A_{1}$, together with the action of $A_{1}$ on $A_{2}$, is the free crossed module on a map $\partial_{2}^{0}: C_{2} \rightarrow A_{1}$, where $C_{2}$ is some set.

3. If $n \geqslant 3$ then $A_{n}$ is the free $\mathbb{Z}(A)$-module on a set $C_{n}$. In particular, $\partial_{n}$ is determined in the obvious way from a certain map $\partial_{n}^{0}: C_{n} \rightarrow A_{n-1}$.

Notice that the action of an $a \in A_{1}$ on $\operatorname{ker}\left(\partial_{2}\right) \subset A_{2}$ depends only on the projection $p(a)$ of $a$ in $\operatorname{coker}\left(\partial_{2}\right)=A$ by the second condition of the definition of crossed complexes.

The sets $C_{n}, n \in \mathbb{N}$ will collectively be called a basis of $\mathcal{A}$.

Let $M$ be a CW-complex with a unique 0-cell, which we take to be its base point. Then $\Pi(M)$ is a totally free crossed complex. This is proved, for example, in $[\mathbf{3 7}, \mathbf{3 8}$, 5], and is a consequence of the relative Hurewicz Theorem, as well as Whitehead's Theorem 2.15. Let us be more explicit. For a more careful treatment of these issues we refer the reader to [4]. If $n \in \mathbb{N}$, we can take each $C_{n}$ to be the set $\left\{e_{1}^{n}, \ldots, e_{l_{n}^{M}}^{n}\right\}$ of $n$-cells of $M$. Each of these $n$-cells attaches to $M^{n-1}$ along an element $\partial\left(e_{i_{n}}^{n}\right)^{n} \in$ $\pi_{n-1}\left(M^{n-1}, *\right), i_{n}=1, \ldots, l_{n}^{M}$, defined up to acting by an element of $\pi_{1}\left(M^{1}, *\right)$. Therefore, if $n>2$, then $\partial_{n}^{0}$ is the image of this element on $\pi_{n-1}\left(M^{n-1}, M^{n-2}, *\right)$. It is well known that $\pi_{n}\left(M^{n}, M^{n-1}, *\right)$ is the free $\mathbb{Z}\left(\pi_{1}\left(M^{2}, *\right)\right)$-module on the set of $n$-cells of $M$, by the relative Hurewicz Theorem. See [34, Chapter V] or [5], for 
example. We can see that for dimensions higher than 3 , a lot of information about the $\mathrm{CW}$-complex $M$ is lost through passing to the fundamental crossed complex of its skeletal filtration.

If $n=2$, then $C_{2}$ is the set of 2-cells of $M$, and by Whitehead's Theorem, the crossed module $\Pi_{2}\left(M^{2}, M^{1}, *\right)$ is the free crossed module on the attaching maps $\partial_{2}^{0}: C_{2} \rightarrow \pi_{1}\left(M^{1}, *\right)$, defined up to conjugation by an element of $\pi_{1}\left(M^{1}, *\right)$.

We have:

Lemma 2.17. Let $M$ be a $C W$-complex with a unique 0 -cell which we take to be its base point $*$. Let $C_{n}$ be the set of cells of $M$ of dimension $n$, where $n \in \mathbb{N}$. For each $e^{n} \in C_{n}$, choose an element of $\pi_{n-1}\left(M^{n-1}, *\right)$ (defined up to acting by an element of $\pi_{1}\left(M^{1}\right)$ ) along which $e^{n}$ attaches to $M^{n-1}$, therefore defining maps $\partial_{n}^{0}: C_{n} \rightarrow$ $\pi_{n-1}\left(M^{n-1}, M^{n-2}, *\right), n \geqslant 3$ and $\partial_{2}^{0}: C_{2} \rightarrow \pi_{1}\left(M^{1}, *\right)$. These choices also define identifications $F\left(C_{n}\right) \rightarrow \pi_{n}\left(M^{n}, M^{n-1}, *\right)$ and $F\left(C_{1}\right) \rightarrow \pi_{1}\left(M^{1}, *\right)$. Here $F\left(C_{n}\right)$ is the free $\mathbb{Z}\left(\pi_{1}(M)\right)$-module on $C_{n}$, if $n>3, F\left(C_{2}\right)$ is the principal group of the free crossed module on $\partial_{2}^{0}: C_{2} \rightarrow \pi_{1}\left(M^{1}, *\right)$, and $F\left(C_{1}\right)$ is the free group on $C_{1}$.

Let $\mathcal{A}=\left(A_{n}, \partial_{n}\right)$ be a (reduced) crossed complex. There exists a one-to-one correspondence between crossed complex morphisms $\Pi(M) \rightarrow \mathcal{A}$ and sequences of maps $f_{n}: C_{n} \rightarrow A_{n}$ such that $f_{n-1} \circ \partial_{0}^{n}=\partial_{n} \circ f_{n}$ for any $n \in \mathbb{N}$.

2.2.2. The cotruncation functor

Let $M$ be a CW-complex. As usual, for any $k \in \mathbb{N}$, let $M^{k}$ denote the $k$-skeleton of $M$. Suppose that $M$ has a unique 0 -cell, which we take to be its base point $*$. Let $L>1$. Note that from the Cellular Approximation Theorem we have

$$
\Pi_{L}\left(M, M^{L-1}, M^{L-2}, \ldots, *\right) \cong \Pi_{L}\left(M^{L+1}, M^{L-1}, M^{L-2}, \ldots, *\right) .
$$

Consider the fundamental crossed complex

$$
\cdots \stackrel{\partial_{4}}{\longrightarrow} \pi_{3}\left(M^{3}, M^{2}, *\right) \stackrel{\partial_{3}}{\longrightarrow} \pi_{2}\left(M^{2}, M^{1}, *\right) \stackrel{\partial_{2}}{\longrightarrow} \pi_{1}\left(M^{1}, *\right) \stackrel{p}{\rightarrow} \pi_{1}(M, *) \rightarrow\{1\}
$$

of the skeletal filtration of $M$. The following is well known:

Lemma 2.18. Let $L>1$. We have:

$$
\pi_{L}\left(M, M^{L-1}, *\right)=\pi_{L}\left(M^{L}, M^{L-1}, *\right) / \operatorname{im}\left(\partial_{L+1}\right) .
$$

This lemma can also be shown using the Higher Homotopy van Kampen Theorem (see Theorem 2.21).

Proof. This follows from the homotopy sequence of $\left(M^{L+1}, M^{L}, M^{L-1}\right)$. Indeed, the following sequence is exact:

$$
\begin{aligned}
\cdots \rightarrow \pi_{L+1}\left(M^{L+1}, M^{L}, *\right) & \stackrel{\partial_{L+1}}{\longrightarrow} \pi_{L}\left(M^{L}, M^{L-1}, *\right) \rightarrow \\
& \rightarrow \pi_{L}\left(M^{L+1}, M^{L-1}, *\right) \rightarrow \pi_{L}\left(M^{L+1}, M^{L}, *\right) \rightarrow \cdots
\end{aligned}
$$

and, moreover, $\pi_{L}\left(M^{L+1}, M^{L}, *\right) \cong\{0\}$. Recall that we have $\pi_{L}\left(M, M^{L-1}, *\right)=$ $\pi_{L}\left(M^{L+1}, M^{L-1}, *\right)$. 
For any $L \in \mathbb{N}$, there exists a cotruncation functor $\operatorname{cotrunc}_{L}$ from the category of crossed complexes to the category of $L$-truncated crossed complexes. If $\mathcal{A}=$ $\left(A_{n}, \partial_{n}, \triangleright_{n}\right)$ is a crossed complex, then $\operatorname{cotrunc}_{L}(\mathcal{A})$ is equal to $\mathcal{A}$ up to dimension $L-1$, whereas $A_{L}$ is substituted by $A_{L} / \operatorname{im}\left(\partial_{L+1}\right)$. Therefore, we can rephrase our previous lemma as:

Proposition 2.19. Let $M$ be a $C W$-complex with a unique 0-cell, which we take to be its base point. Let $L \in \mathbb{N}$. We have:

$$
\Pi_{L}\left(M, M^{L-1}, M^{L-2}, \ldots, *\right)=\operatorname{cotrunc}_{L}(\Pi(M)) .
$$

2.2.3. Colimit theorems: the Higher Homotopy van Kampen Theorem Let $M$ be a connected space equipped with a filtration $\left\{M_{n}\right\}_{n \in \mathbb{N}}$.

Definition 2.20. We say that $\left\{M_{n}\right\}_{n \in \mathbb{N}}$ is connected if all the spaces $M_{n}, n \in \mathbb{N}$ are connected, and, moreover, $\pi_{n}\left(M_{k}, M_{n}, *\right)=0$ if $k \geqslant n$, for any $n \in \mathbb{N}$.

Therefore, if $M$ is a connected $\mathrm{CW}$-complex with a unique 0-cell, then its skeletal filtration is connected. Similarly, if $L>1$ is an integer, then any filtration of the type $\left(M, M^{L}, M^{L-1}, \ldots, M^{0}\right)$ is connected as well. The following beautiful theorem is due to R. Brown and P.J. Higgins. See $[\mathbf{1 0 , ~ 1 1 , ~ 1 4 , ~ 5 , ~ 8 ] . ~ W e ~ w i l l ~ r e f e r ~ t o ~ i t ~ a s ~}$ the "Higher Homotopy van Kampen Theorem".

Theorem 2.21 (R. Brown and P.J. Higgins). Let $M=\left\{M_{n}\right\}$ and $N=\left\{N_{n}\right\}$ be filtered spaces such that $M_{0}$ and $N_{0}$ are singletons, taken to be the base points of $M$ and $N$. Let $U \subset M$, and let $i: U \rightarrow M$ be the inclusion map. Let $U$ inherit the filtration $\left\{U_{n}\right\}$ induced by the filtration of $M$. Suppose that the filtrations of $M, N$ and $U$ are connected. In addition, we suppose that the inclusions $i_{n} \doteq i_{U_{n}}: U_{n} \rightarrow$ $M_{n}$ are closed cofibrations, for any $n \in \mathbb{N}$. Let $f: U \subset M \rightarrow N$ be a filtered map. Therefore the adjunction space

$$
V \doteq M \bigcup_{u \in U \mapsto f(u)} N
$$

is naturally filtered by the set

$$
V_{n} \doteq M_{n} \bigcup_{u \in U_{n} \mapsto f(u)} N_{n}, n \in \mathbb{N},
$$

and $V_{0}$ has a unique element. We thus have a commutative diagram of filtered spaces:

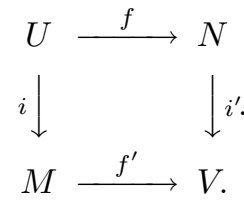

The following hold:

1. The filtered space $V$ is connected. 
2. The diagram

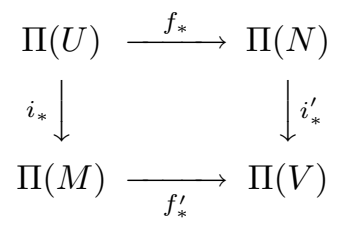

is a pushout of crossed complexes.

This theorem also holds for infinite adjunction spaces. In particular, it follows:

Corollary 2.22. Let $\left\{M_{\lambda}, \lambda \in \Lambda\right\}$ be a family of $C W$-complexes. Suppose that they all have unique 0-cells, which we take to be their base points *. Form the wedge product

$$
\bigvee_{\lambda \in \Lambda}\left(M_{\lambda}, *\right)
$$

along $*$, which is also a $C W$-complex $\bigvee_{\lambda \in \Lambda} M_{\lambda}$, having a unique 0-cell. The obvious map

$$
\bigvee_{\lambda \in \Lambda} \Pi\left(M_{\lambda}\right) \rightarrow \Pi\left(\bigvee_{\lambda \in \Lambda} M_{\lambda}\right),
$$

induced by the inclusions, is an isomorphism of crossed complexes. In addition, if $L \in \mathbb{N}$, the natural map

$$
\bigvee_{\lambda \in \Lambda} \Pi_{L}\left(M_{\lambda}\right) \rightarrow \Pi_{L}\left(\bigvee_{\lambda \in \Lambda} M_{\lambda}\right)
$$

is also an isomorphism.

This is Theorem 7.1 of [10].

Remark 2.23. The fact that the fundamental crossed complex of a CW-complex is totally free (referred to in 2.2.1) is also a corollary of the Higher Homotopy van Kampen Theorem.

\subsubsection{Homotopy of crossed complexes}

The main references here are $[\mathbf{5}, \mathbf{1 1}, \mathbf{1 2}, \mathbf{1 3}, \mathbf{3 8}, \mathbf{3 9}]$. Let $G$ and $G^{\prime}$ be groups. Suppose that $G^{\prime}$ has a left action $\triangleright^{\prime}$ on the group $E^{\prime}$ by automorphisms. Let $\phi: G \rightarrow$ $G^{\prime}$ be a group morphism. A map $s: G \rightarrow E^{\prime}$ is said to be a $\phi$-derivation if

$$
s(X Y)=\left(\phi(Y)^{-1} \triangleright^{\prime} s(X)\right) s(Y), \forall X, Y \in G .
$$

Notice that if $G$ is a free group, then a $\phi$-derivation can be uniquely specified by its value on the set of free generators of $G$. More precisely, any map from the set of free generators of $G$ into $E^{\prime}$ extends uniquely to a $\phi$-derivation, and any $\phi$-derivation arises in this way. This is easy to show, but see [38, Lemma 3], for example.

Definition 2.24. Let $\mathcal{A}=\left(A_{n}, \partial_{n}, \triangleright_{n}\right)$ and $\mathcal{B}=\left(B_{n}, \partial_{n}^{\prime}, \triangleright_{n}^{\prime}\right)$ be crossed complexes, and let $f=\left(f_{n}\right): \mathcal{A} \rightarrow \mathcal{B}$ be a morphism. If $L \geqslant 1$ is a positive integer, an $L$-fold $f$-homotopy is a sequence $H=\left(H_{n}\right)$ of maps $H_{n}: A_{n} \rightarrow B_{n+L}$ such that: 
1. If $n>1$ then $H_{n}$ is a group morphism and moreover

$$
H_{n}\left(a_{1} \triangleright a_{n}\right)=f_{1}\left(a_{1}\right) \triangleright^{\prime} H_{n}\left(a_{n}\right), \forall a_{1} \in A_{1}, \forall a_{n} \in A_{n} .
$$

2. $H_{1}$ is an $f_{1}$-derivation.

A 1-fold $f$-homotopy will be simply called an $f$-homotopy. Note that there are no compatibility relations between the boundary maps of $\mathcal{A}$ and $\mathcal{B}$ and the maps $H_{n}$, where $n \in \mathbb{N}$, whenever $H=\left(H_{n}\right)$ is an $L$-fold $f$-homotopy. Note also that the notion of an $L$-fold $f$-homotopy depends only on $f_{1}: A_{1} \rightarrow B_{1}$ and the actions of $A_{1}$ in $A_{n}$ and $B_{1}$ on $B_{n}$, where $n \in 2,3, \ldots$.

We have (see $[\mathbf{3 8}, \mathbf{5}, \mathbf{1 2}])$ :

Lemma 2.25. Under the conditions of the previous definition, if $H$ is an $f$-homotopy, where $f: \mathcal{A} \rightarrow \mathcal{B}$, then the sequence of maps $g=\left(g_{n}\right)$ such that

$$
g_{n}\left(a_{n}\right)=f_{n}\left(a_{n}\right)\left(H_{n-1} \circ \partial\right)\left(a_{n}\right)\left(\partial^{\prime} \circ H_{n}\right)\left(a_{n}\right), a_{n} \in A_{n}, n \geqslant 2
$$

and

$$
g_{1}\left(a_{1}\right)=f_{1}\left(a_{1}\right)\left(\partial^{\prime} \circ H_{1}\right)\left(a_{1}\right), a_{1} \in A_{1}
$$

is a morphism of crossed complexes, in which case we say that $H$ realises a homotopy $f \stackrel{H}{\longrightarrow} g$.

We can consider the category of crossed complexes with homotopy classes of morphisms of crossed complexes as morphisms. The following result is due to J.H.C. Whitehead, see [38, Theorem 5], and gives us one answer as to how $\Pi(M)$ depends on the $\mathrm{CW}$-decomposition of $M$, if $M$ is a CW-complex.

Theorem 2.26 (J.H.C. Whitehead). Let $M$ and $N$ be $C W$-complexes with a unique 0-cell. Let F, $G: M \rightarrow N$ be homotopic cellular maps. Then the induced maps $F_{*}, G_{*}: \Pi(M) \rightarrow \Pi(N)$ are homotopic. In particular, if $M$ and $N$ are homotopic (as spaces) then it follows that $\Pi(M)$ and $\Pi(N)$ have the same homotopy type.

This result can be proved using R. Brown and P.J. Higgins's framework on $\omega$ groupoids and the tensor product of crossed complexes, and is, ultimately, a consequence of the Cellular Approximation Theorem. See $[\mathbf{1 2}, \mathbf{1 3}]$. It is not difficult to show directly that a homotopy $H: F \rightarrow G$, chosen to be cellular, will induce a crossed complex homotopy $F_{*} \rightarrow G_{*}$.

Remark 2.27. In fact, Whitehead's results imply a stronger statement: Suppose that $M$ and $N$ are finite of dimension $\leqslant L$. Then $\Pi\left(M^{\prime}\right)$ and $\Pi\left(N^{\prime}\right)$ are simply homotopy equivalent. Here $M^{\prime}$ and $N^{\prime}$ are obtained from $M$ and $N$ by making a wedge product with a certain finite number of spheres $S^{L}$. This is a consequence of Theorem 16 of [39]. For the definition of simple homotopy equivalence of crossed complexes, we refer the reader to $[\mathbf{3 9}]$.

One of our main results (Theorem 4.1) extends (J.H.C. Whitehead's) Theorem 2.26 in a direction very similar to the one indicated in the previous remark. 


\subsubsection{The definition of $C R S(\mathcal{A}, \mathcal{B})$}

Given crossed complexes $\mathcal{A}$ and $\mathcal{B}$, there exists a groupoid $C R S_{1}(\mathcal{A}, \mathcal{B})$ whose objects are the morphisms of crossed complexes $\mathcal{A} \rightarrow \mathcal{B}$, and whose morphisms are the homotopies connecting them, in the manner shown in Lemma 2.25, with pointwise product of maps as composition. We denote the set of all $f$-homotopies by $C R S_{1}^{f}(\mathcal{A}, \mathcal{B})$. Therefore $C R S_{1}^{f}(\mathcal{A}, \mathcal{B})$ is the set of morphisms of $C R S_{1}(\mathcal{A}, \mathcal{B})$ with source $f \in \operatorname{Hom}(\mathcal{A}, \mathcal{B})$.

In fact, a far stronger result is true: Let $\mathcal{A}$ and $\mathcal{B}$ be crossed complexes, and let $f=\left(f_{n}\right): \mathcal{A} \rightarrow \mathcal{B}$ be a morphism. Let $n \geqslant 2$. An $n$-fold $f$-homotopy $H$ determines naturally a $(n-1)$-fold $f$-homotopy $\partial_{n}^{f}(H)$, in the same way that a 1 -fold $f$-homotopy determines a morphism $\mathcal{A} \rightarrow \mathcal{B}$. Let $C R S_{n}^{f}(\mathcal{A}, \mathcal{B})$ be the set of $n$-fold $f$-homotopies. It is a group with pointwise multiplication as product. In particular, for any $n>1$,

$$
C R S_{n}(A, B)=\bigcup_{f \in \operatorname{Hom}(\mathcal{A}, \mathcal{B})} C R S_{n}^{f}(\mathcal{A}, \mathcal{B})
$$

is a totally disconnected groupoid with the same object set as $C R S_{1}(\mathcal{A}, \mathcal{B})$. There exist also natural left groupoid actions of $C R S_{1}(\mathcal{A}, \mathcal{B})$ on $C R S_{n}(\mathcal{A}, \mathcal{B})$, for any $n \geqslant 2$. The following theorem is shown in $[\mathbf{5}, \mathbf{1 1}, \mathbf{1 2}, \mathbf{1 3}]$, where the details of this description can be found.

Theorem 2.28 (R. Brown and P.J. Higgins). Let $\mathcal{A}$ and $\mathcal{B}$ be (reduced) crossed complexes. Then the sequence $C R S(\mathcal{A}, \mathcal{B})=\left(C R S_{n}(\mathcal{A}, \mathcal{B})\right)$ can be given the structure of a (non-reduced) crossed complex.

Once again this is part of a much more general theory. There also exists a tensor product in the category of crossed complexes, making it a monoidal closed category; see $[\mathbf{1 2}]$.

Remark 2.29. Actually R. Brown and P.J. Higgins considered a more general notion of homotopy (free homotopy), also considered in [38]. The restricted notion of homotopy shown here was called pointed homotopy by them. We will not need to consider the full generality.

\subsubsection{Counting homotopies}

The following lemma will be extremely useful. It is due to the fact that there are no constraint relations between a homotopy and the boundary maps of the crossed complexes involved. It appears in [12].

Lemma 2.30. Let $\mathcal{F}=\left(F_{n}, \partial_{n}\right)$ be a totally free (reduced) crossed complex. Let $C_{n}$ denote the set of free generators of $F_{n}, n=1,2, \ldots$, as a free group if $n=1$, as a free crossed module if $n=2$, and as a free $\mathbb{Z}\left(\operatorname{coker}\left(\partial_{2}\right)\right)$-module if $n>2$. Let $\mathcal{A}=\left(A_{n}, \partial_{n}^{\prime}\right)$ be a (reduced) crossed complex, and let $f: \mathcal{F} \rightarrow \mathcal{A}$ be a crossed complex morphism. An L-fold $f$-homotopy can be specified, uniquely, by its value on each set $C_{n}, n \in \mathbb{N}$, in the sense that any sequence of maps $C_{n} \rightarrow A_{n+L}$ uniquely extends to an L-fold $f$-homotopy, and any L-fold crossed complex homotopy arises in this way. 
Proof. The only bit that needs discussion is the definition of $H_{2}: F_{2} \rightarrow A_{L+2}$, from the known form of $\mathrm{H}_{2}$ restricted to $\mathrm{C}_{2}$. This follows from the universal property defining free crossed modules (Definition 2.14), applied to the crossed module $A_{2+L} \stackrel{a \mapsto 1}{\longrightarrow} A=\operatorname{coker}\left(\partial_{2}^{\prime}\right)$. Note condition (4) of the definition of crossed complexes.

Therefore:

Corollary 2.31. Let $M$ be a $C W$-complex with a unique 0-cell, which we take to be its base point. Also let $\mathcal{A}$ be a reduced crossed complex, and let $f: \Pi(M) \rightarrow \mathcal{A}$ be a morphism of crossed complexes. An n-fold f-homotopy is uniquely specified by its value on each cell of $M$, in the sense shown above. In fact, if $\mathcal{A}$ is L-truncated, then an $n$-fold homotopy $\Pi_{L}(M)$ to $M$ is also uniquely specified by its value on the cells of $M$ of dimension $1,2, \ldots, L-1$.

The following simple quantification of the previous corollary will have an important role later:

Corollary 2.32. Let $M$ be a $C W$-complex with a unique 0-cell, which we take to be its base point, and a finite number of $n$-cells for any $n \in \mathbb{N}$. Also let $\mathcal{A}=\left(A_{n}\right)$ be a (reduced) crossed complex, which we suppose to be finite, in the sense that $\mathcal{A}$ is l-truncated, for some $l \in \mathbb{N}$, and each $A_{n}, n \in \mathbb{N}$ is finite. Then $C R S(\Pi(M), \mathcal{A})$ is a finite crossed complex and, moreover, for any $L \in \mathbb{N}$ and any $f \in \operatorname{Hom}(\Pi(M), \mathcal{A})$ we have

$$
\#\left(C R S_{L}^{f}(\Pi(M), \mathcal{A})\right)=\prod_{k=1}^{\infty}\left(\#\left(A_{k+L}\right)\right)^{l_{k}^{M}},
$$

where $l_{k}^{M}$ denotes the number of cells of $M$ of order $k \in \mathbb{N}$.

\subsubsection{The classifying space of a crossed complex}

There exists a functor, the classifying space functor, from the category of (reduced or non-reduced) crossed complexes to the category of filtered spaces. This functor, due to R. Brown and P.J. Higgins, was described in [13]. It generalises the concept of the classifying space of a group. A similar construction of classifying spaces of crossed complexes appeared in $[\mathbf{3}]$.

For a crossed complex $\mathcal{A}=\left(A_{n}\right)$, let $|\mathcal{A}|$ denote its classifying space. It is a CWcomplex, with one 0 -cell for each element of $C$, where $C$ is the set of objects of the groupoid $A_{1}$. A very strong result appearing in $[\mathbf{1 3}]$ is the following one:

Theorem 2.33 (R. Brown and P.J. Higgins). Let $M$ be a $C W$-complex, provided with its skeletal filtration. Suppose $M$ has a unique 0-cell which we take to be its base point. Also let $\mathcal{A}$ be a reduced crossed complex; thus $|\mathcal{A}|$ has a unique 0 -cell $*$. There exists a map

$$
\psi:|C R S(\Pi(M), \mathcal{A})| \underset{\simeq}{\longrightarrow} \operatorname{TOP}((M, *),(|\mathcal{A}|, *)),
$$

which is a weak homotopy equivalence. 
In particular, if $M$ is a finite CW-complex, then $\psi$ is a homotopy equivalence. This is because in this case the function space $\operatorname{TOP}((M, *),(|\mathcal{A}|, *))$ has the homotopy type of a CW-complex. See [27].

The proof of Theorem 2.33 which appears in [13] makes great use of the monoidal closed structure of the category of crossed complexes constructed in $[\mathbf{1 2}]$. A version of the Eilenberg-Zilber Theorem for crossed complexes, representing $\Pi(|C \times D|)$ as a strong deformation retract of $\Pi(|C|) \otimes \Pi(|D|)$, if $C$ and $D$ are simplicial sets, is also required. An approach to this is sketched in [13]. A direct proof appears in $[32,33]$. A theorem of J. Milnor establishing that a Kan Complex is a strong deformation retract of the singular complex of its geometric realisation is implicitly used; see [28].

Let $\mathcal{A}$ be a crossed complex. We will now consider the full generality in the definition of crossed complexes, as sketched in Remark 2.13. Let $C$ be the object set of the groupoid $A_{1}$. Denote by $A_{n}^{c}$ the set of morphisms of $A_{n}$ whose source is $c$. Therefore $A_{n}^{c}$ is a group if $n>1$. Also let $A_{1}^{(c, d)}$ be the set of morphisms of $A_{1}$ with source and target $c, d \in C$, respectively. Let $\partial_{n}^{c}=\partial_{n \mid A_{n}^{c}}$. Consider the complex $\mathcal{A}^{c}=$ $\left(A_{n}^{c}, \partial_{n}^{c}\right), n>1$, considering the group $\mathcal{A}_{1}^{c, c}$ at index 1 . Define $\pi_{1}(\mathcal{A}, c)=H_{1}\left(\mathcal{A}^{c}\right)$, and $H_{n}(\mathcal{A}, c)=H_{n}\left(\mathcal{A}^{c}\right)$ if $n \geqslant 2$. Notice that these homotopy and homology groups with base $c \in C$ depend only on the connected component in the groupoid $A_{1}$ to which $c$ belongs, up to isomorphism.

If $M$ is a CW-complex with a unique 0-cell, then $\pi_{1}(M, *) \cong \pi_{1}(\Pi(M), *)$. On the other hand, $H_{n}(\hat{M}) \cong H_{n}(\Pi(M))$, for $n \geqslant 2$. Here $\hat{M}$ is the universal covering of $M$.

Theorem 2.34 (R. Brown and P.J. Higgins). Let $\mathcal{A}$ be a crossed complex. Let $C$ be the set of objects of the groupoid $A_{1}$. Then the classifying space $|\mathcal{A}|$ of $\mathcal{A}$ is a $C W$-complex with one 0 -cell c for each element of $c \in C$. Moreover,

$$
\begin{aligned}
\pi_{1}(|\mathcal{A}|, c) \cong \pi_{1}(\mathcal{A}, c) \\
\pi_{n}(|\mathcal{A}|, c) \cong H_{n}(\mathcal{A}, c), n \geqslant 2,
\end{aligned}
$$

for any $c \in C$. Furthermore, there exists a one-to-one correspondence between connected components of the groupoid $A_{1}$ and connected components of $|\mathcal{A}|$.

\section{The homotopy type of the skeletal filtration of a CW- complex}

Let $M$ be a space which can be given a CW-complex structure. In this chapter we analyse to what extent the homotopy type of the skeletal filtration $\left\{M^{n}\right\}_{n \in \mathbb{N}}$ of $M$ depends on the cellular decomposition of $M$.

Throughout this chapter, $D^{n}$ denotes the $n$-disk and $S^{n}$ denotes the $n$-sphere. As usual, we set $I=[0,1]$.

\subsection{Dimension two}

Let $(N, M)$ be a pair of CW-complexes such that the inclusion of $M$ in $N$ is a homotopy equivalence. For simplicity, assume that $M$ and $N$ have a finite number 
of 1-cells. Our whole discussion will remain valid without this restriction, with the obvious modifications.

Let $M^{1}$ and $N^{1}$ be, respectively, the 1-skeletons of $M$ and $N$. Suppose that $N$ and $M$ have a unique 0 -cell, which we take to be their common base point $*$, so that both $M$ and $N$ are well pointed.

The group $\pi_{1}\left(M^{1}, *\right)$ is the free group on the set $\left\{X_{1}, \ldots, X_{m}\right\}$ of 1-cells of $M$. Let $Y_{1}, \ldots, Y_{n}$ be the 1-cells of $N$ which are not in $M$. Then $\pi_{1}\left(N^{1}, *\right)$ is the free group $F\left(X_{1}, \ldots, X_{m}, Y_{1}, \ldots, Y_{n}\right)$ on the set $\left\{X_{1}, \ldots, X_{m}, Y_{1}, \ldots, Y_{n}\right\}$.

Theorem 3.1. There exists a filtered homotopy equivalence

$$
\left(N, N^{1}, *\right) \cong\left(M, M^{1}, *\right) \vee\left(D^{2}, S^{1}, *\right)^{\vee n} .
$$

Proof. ${ }^{3}$ Since $M$ is a subcomplex of $N$, and $N$ is homotopic to $M$, it follows that $M$ is a strong deformation retract of $N$. By the Cellular Approximation Theorem, we can suppose, furthermore, that there exists a retraction $r: N \rightarrow M$ sending $N^{1}$ to $M^{1}$, and such that $r \cong \operatorname{id}_{N}$, relative to $M$. In particular, if $k \in\{1, \ldots, n\}$ then we have $Y_{k} r_{*}\left(Y_{k}\right)^{-1}=1_{\pi_{1}(N, *)}$ (though this relation does not hold in $\pi_{1}\left(N^{1}, *\right)$ ). Define a map

$$
f:\left(P, P^{1}, *\right) \doteq\left(M, M^{1}, *\right) \vee \bigvee_{k=1}^{n}\left(D_{k}^{2}, S_{k}^{1}, *\right) \rightarrow\left(N, N^{1}, *\right)
$$

in the following way: First of all, send $\left(M, M^{1}, *\right)$ identically to its copy $\left(M, M^{1}, *\right) \subset$ $\left(N, N^{1}, *\right)$. Then we can send each $\left(S_{k}^{1}, *\right), k=1, \ldots, n$ to the element $Y_{k} r_{*}\left(Y_{k}\right)^{-1} \in$ $\pi_{1}\left(N^{1}, *\right)$. Since these elements are null homotopic in $(N, *)$, this map extends to the remaining 2-cells of $\left(P, P^{1}, *\right)$.

Let us prove that $f:\left(P, P^{1}, *\right) \rightarrow\left(N, N^{1}, *\right)$ is a homotopy equivalence. It suffices to prove that $f:(P, *) \rightarrow(N, *)$ and $f^{1} \doteq f_{\mid P^{1}}:\left(P^{1}, *\right) \rightarrow\left(N^{1}, *\right)$ are based homotopy equivalences, by Theorem 2.3 .

We first show that $f$ is a homotopy equivalence $(P, *) \rightarrow(N, *)$. Let $r^{\prime}:(P, *) \rightarrow$ $(M, *)$ be the obvious retraction, thus $r^{\prime} \cong \operatorname{id}_{(P, *)}$. We have $r \circ f \cong r \circ f \circ r^{\prime}=r^{\prime} \cong$ $\operatorname{id}_{(P, *)}$, and $f \circ r=r \cong \operatorname{id}_{(N, *)}$.

We now show that $f^{1}$ is a homotopy equivalence $\left(P^{1}, *\right) \rightarrow\left(N^{1}, *\right)$. It is enough to prove that the induced map $f_{*}^{1}: \pi_{1}\left(P^{1}, *\right) \rightarrow \pi_{1}\left(N^{1}, *\right)$ is an isomorphism. Note that $\pi_{1}\left(P^{1}, *\right)$ is (similar to $\pi_{1}\left(N^{1}, *\right)$ ) isomorphic to the free group on the set $\left\{X_{1}, \ldots, X_{m}, Y_{1}, \ldots, Y_{n}\right\}$. The induced map on the fundamental groups has the form

$$
f_{*}^{1}\left(X_{k}\right)=X_{k}, k=1, \ldots, m, \text { and } f_{*}^{1}\left(Y_{k}\right)=Y_{k} r_{*}\left(Y_{k}\right)^{-1}, k=1, \ldots, n .
$$

Notice that $r_{*}\left(Y_{k}\right) \in F\left(X_{1}, \ldots, X_{m}\right), k=1, \ldots, n$. Consider the morphism $g$ of the

\footnotetext{
${ }^{3}$ This argument arose in a discussion with Gustavo Granja.
} 
group $F\left(X_{1}, \ldots, X_{m}, Y_{1}, \ldots, Y_{n}\right)$ on itself such that

$$
g\left(X_{k}\right)=X_{k}, k=1, \ldots, m \text { and } g\left(Y_{k}\right)=Y_{k} r_{*}\left(Y_{k}\right), k=1, \ldots, n .
$$

Therefore $\left(f_{*}^{1} \circ g\right)\left(X_{k}\right)=X_{k}, k=1, \ldots, m$, and

$$
\begin{aligned}
\left(f_{*}^{1} \circ g\right)\left(Y_{k}\right) & =f_{*}^{1}\left(Y_{k} r_{*}\left(Y_{k}\right)\right) \\
& =f_{*}^{1}\left(Y_{k}\right) f_{*}^{1}\left(r_{*}\left(Y_{k}\right)\right) \\
& =Y_{k} r_{*}\left(Y_{k}\right)^{-1} r_{*}\left(Y_{k}\right) \\
& =Y_{k}, k=1, \ldots, n .
\end{aligned}
$$

Analogously, $\left(g \circ f_{*}\right)\left(X_{k}\right)=X_{k}, k=1, \ldots, m$, and

$$
\begin{aligned}
\left(g \circ f_{*}^{1}\right)\left(Y_{k}\right) & =g\left(Y_{k} r_{*}\left(Y_{k}\right)^{-1}\right) \\
& =Y_{k} r_{*}\left(Y_{k}\right) g\left(r_{*}\left(Y_{k}^{-1}\right)\right) \\
& =Y_{k} r_{*}\left(Y_{k}\right) r_{*}\left(Y_{k}^{-1}\right)=Y_{k}, k=1, \ldots, n .
\end{aligned}
$$

This proves that $g^{-1}=f_{*}^{1}$, which finishes the proof.

Corollary 3.2. Let $M$ and $N$ be $C W$-complexes with unique 0 -cells, which we take to be their base points, both denoted by $*$. Let $m_{1}$ and $n_{1}$ be the number of 1-cells of $M$ and $N$, respectively. Suppose that $M$ and $N$ are based homotopic, as spaces. There exists a filtered homotopy equivalence

$$
f:\left(M, M^{1}, *\right) \vee\left(D^{2}, S^{1}, *\right)^{\vee n_{1}} \rightarrow\left(N, N^{1}, *\right) \vee\left(D^{2}, S^{1}, *\right)^{\vee m_{1}} .
$$

Moreover, $f$ can be chosen cellular.

Proof. Let $F: M \rightarrow N$ be a pointed homotopy equivalence. We can suppose that $F$ is a cellular map. The reduced mapping cylinder $P$ of $F$ is a $C W$-complex with a unique 0 -cell, containing $M$ and $N$ as subcomplexes. Moreover $P$ is homotopic to both $M$ and $N$. The complex $P$ has $n_{1}$ 1-cells which are not in $M$ and $m_{1}$ 1-cells which are not in $N$. By the previous theorem, it thus follows that there exists a filtered homotopy equivalence

$$
F^{\prime}: \mathcal{M} \doteq\left(M, M^{1}, *\right) \vee\left(D^{2}, S^{1}, *\right)^{\vee n_{1}} \rightarrow\left(N, N^{1}, *\right) \vee\left(D^{2}, S^{1}, *^{\prime}\right)^{\vee m_{1}} \doteq \mathcal{N} .
$$

Let $f$ be a map, homotopic to $F^{\prime}$, which is cellular. We can suppose that $F$ coincides with $F^{\prime}$ in $\left(M^{1}, *\right) \vee\left(S^{1}, *\right)^{\vee n_{1}}=\left(\mathcal{M}^{1}, *\right)$. Then $f$ is a homotopy equivalence $f: \mathcal{M} \rightarrow \mathcal{N}$, and, therefore, by Theorem 2.3, $f$ is a filtered homotopy equivalence.

\subsection{The general case}

Let $M$ and $N$ be CW-complexes, homotopic as (based) spaces. For simplicity, we suppose that they only have a finite number of cells at each dimension. The main results that we obtain in this subsection (Theorems 3.3, 3.4 and 3.5) are valid without this restriction, although they need to be re-written in the natural way. The proof we will give will remain valid, as long the obvious modifications are made. As 
usual, we suppose $M$ and $N$ to have a unique 0 -cell, which we choose to be the base points of each, so that $M$ and $N$ are well pointed.

For a $k \in \mathbb{N}$, let $l_{k}^{M}$ and $l_{k}^{N}$ be the number of cells of $M$ and $N$ (respectively) of dimension $k$. Define the integer numbers

$$
\begin{aligned}
& l_{\{M, N\}}^{M}(1)=l_{1}^{N}, \\
& l_{\{M, N\}}^{N}(1)=l_{1}^{M} .
\end{aligned}
$$

And, in general, if $k>1$,

$$
\begin{aligned}
& l_{\{M, N\}}^{M}(k+1)=l_{\{M, N\}}^{N}(k)+l_{k+1}^{N}, \\
& l_{\{M, N\}}^{N}(k+1)=l_{\{M, N\}}^{M}(k)+l_{k+1}^{M} .
\end{aligned}
$$

Define, for each $L \geqslant 2$, the filtered space

$$
\begin{gathered}
\mathcal{M}_{L}=\left(M, M^{L-1}, \ldots, M^{1}, *\right) \vee \bigvee_{j_{(L-1)}=1}^{l_{\{M, N\}}^{M}(L-1)}\left(D_{j_{(L-1)}}^{L}, S_{j_{(L-1)}}^{(L-1)}, *, \ldots, *\right) \vee \\
\vee \bigvee_{j_{(L-2)}=1}^{l_{\{M, N\}}^{M}(L-2)}\left(D_{j_{(L-2)}}^{(L-1)}, D_{j_{(L-2)}}^{(L-1)}, S_{j_{(L-2)}}^{(L-2)}, *, \ldots, *\right) \vee \cdots \\
\cdots \vee \bigvee_{j_{2}=1}^{l_{\{M, N\}}^{M}(2)}\left(D_{j_{2}}^{3}, \ldots, D_{j_{2}}^{3}, S_{j_{2}}^{2}, *, *\right) \vee \bigvee_{j_{1}=1}^{l_{\{M, N\}}^{M}(1)}\left(D_{j_{1}}^{2}, \ldots, D_{j_{1}}^{2}, S_{j_{1}}^{1}, *\right) .
\end{gathered}
$$

Analogously, define

$$
\begin{gathered}
\mathcal{N}_{L}=\left(N, N^{L-1}, \ldots, N^{1}, *\right) \vee \bigvee_{i_{(L-1)}=1}^{l_{\{M, N\}}^{N}(L-1)}\left(D_{i_{(L-1)}}^{L}, S_{i_{(L-1)}}^{(L-1)}, *, \ldots, *\right) \vee \\
\vee \bigvee_{i_{(L-2)}=1}^{l_{\{M, N\}}^{N}(L-2)}\left(D_{i_{(L-2)}}^{(L-1)}, D_{i_{(L-2)}}^{(L-1)}, S_{i_{(L-2)}}^{(L-2)}, *, \ldots, *\right) \vee \cdots \\
\cdots \vee \bigvee_{i_{2}=1}^{l_{\{M, N\}}^{N}(2)}\left(D_{i_{2}}^{3}, \ldots, D_{i_{2}}^{3}, S_{i_{2}}^{2}, *, *\right) \vee \bigvee_{i_{1}=1}^{l_{\{M, N\}}(1)}\left(D_{i_{1}}^{2}, \ldots, D_{i_{1}}^{2}, S_{i_{1}}^{1}, *\right)
\end{gathered}
$$

Therefore $\mathcal{M}_{L}$ (as a CW-complex) has $l_{L}^{M}+l_{\{M, N\}}^{M}(L-1)=l_{\{M, N\}}^{N}(L)$ cells of dimension $L$, and, analogously, $\mathcal{N}_{L}$ has $l_{L}^{N}+l_{\{M, N\}}^{N}(L-1)=l_{\{M, N\}}^{M}(L)$ cells of dimension $L$, for $L=2,3, \ldots$. Define also $\mathcal{M}_{1}=(M, *)$ and $\mathcal{N}_{1}=(N, *)$.

For each positive integer $L$, the $\mathrm{CW}$-complex $\mathcal{M}_{L}$ is embedded cellularly in 
$\mathcal{M}_{L+1}$, and analogously for $\mathcal{N}_{L}$. Therefore the spaces

$$
\begin{aligned}
\mathcal{M} & =\bigcup_{L=1}^{\infty} \mathcal{M}_{L} \\
& =M \vee \bigvee_{L=1}^{\infty}\left(\bigvee_{i_{L}=1}^{l_{\{M, N\}}^{M}(L)} D_{i_{L}}^{L+1}\right)
\end{aligned}
$$

and

$$
\begin{aligned}
\mathcal{N} & =\bigcup_{L=1}^{\infty} \mathcal{N}_{L} \\
& =N \vee \bigvee_{L=1}^{\infty}\left(\bigvee_{j_{L}=1}^{l_{\{M, N\}}^{N}(L)} D_{j_{L}}^{L+1}\right)
\end{aligned}
$$

are $\mathrm{CW}$-complexes. Filter $\mathcal{M}$ and $\mathcal{N}$ by their skeletal filtrations. Notice that we regard each $(L+1)$-disk $D^{L+1}$, where $L \in \mathbb{N}$, as having the obvious cellular decomposition with a unique 0 -cell, an $L$-cell and an $(L+1)$-cell.

It is important to note that the following holds for each $L \in \mathbb{N}$ :

$$
\begin{aligned}
\mathcal{M}^{L-1} & =\mathcal{M}_{L}^{L-1}, \\
\mathcal{N}^{L-1} & =\mathcal{N}_{L}^{L-1} .
\end{aligned}
$$

We want to prove the following theorem:

Theorem 3.3. In the above situation, for any $L \in \mathbb{N}$ there exists a filtered homotopy equivalence

$$
F_{L}: \mathcal{M}_{L} \cong \mathcal{N}_{L}
$$

which can be taken cellular. Moreover, if we are provided with a filtered homotopy equivalence $F_{L}: \mathcal{M}_{L} \rightarrow \mathcal{N}_{L}$ which is a cellular map, then there exists a filtered homotopy equivalence $F_{L+1}: \mathcal{M}_{L+1} \rightarrow \mathcal{N}_{L+1}$ which extends the map $F_{L}^{L-1}$, the restriction of $F_{L}$ to $\mathcal{M}_{L}^{L-1}=\mathcal{M}_{L+1}^{L-1}$.

Since $\mathcal{M}_{L}^{L-1}=\mathcal{M}^{L-1}$ and $\mathcal{N}_{L}^{L-1}=\mathcal{N}^{L-1}$ for any $L \in \mathbb{N}$, it follows (using Corollary 2.9):

Theorem 3.4. Let $M$ and $N$ be $C W$-complexes with unique 0-cells, taken to be their base points. Suppose that $M$ and $N$ are homotopic as (based) spaces. There exists a filtered homotopy equivalence

$$
F: \mathcal{M} \cong \mathcal{N}
$$

The proof of Theorem 3.3 is by an obvious induction on $L$. Notice that we have already proved it for $L=2$, dealt with in Subsection 3.1. The general proof follows immediately from Theorem 3.5 below, and the fact, already stated, that $\mathcal{M}_{L}$ has $l_{L}^{M}+l_{\{M, N\}}^{M}(L-1)=l_{\{M, N\}}^{N}(L)$ cells of dimension $L$, for any $L \in \mathbb{N}$, and analogously for $\mathcal{N}_{L}$. 


\subsubsection{An auxiliary discussion}

Let $M$ and $N$ be CW-complexes. As usual, we suppose that they have unique 0 cells, which we take to be their base points, both denoted by $*$. Suppose that for some $L>1$ there exists a filtered homotopy equivalence

$$
F:\left(M, M^{L-1}, M^{L-2}, \ldots, M^{0}=*\right) \rightarrow\left(N, N^{L-1}, N^{L-2}, \ldots, N^{0}=*\right),
$$

which, furthermore, is a cellular map. Let $m_{L}$ and $n_{L}$ be, respectively, the number of cells of $M$ and $N$ of dimension $L$.

Theorem 3.5. There exists a filtered homotopy equivalence

$$
\begin{aligned}
F^{\prime \prime}:\left(M, M^{L},\right. & \left.M^{L-1}, \ldots, M^{0}=*\right) \vee \bigvee_{i_{L}=1}^{n_{L}}\left(D_{i_{L}}^{L+1}, S_{i_{L}}^{L}, *, \ldots, *\right) \doteq \bar{M} \\
& \rightarrow\left(N, N^{L}, N^{L-1}, \ldots, N^{0}=*\right) \vee \bigvee_{j_{L}=1}^{m_{L}}\left(D_{j_{L}}^{L+1}, S_{j_{L}}^{L}, *, \ldots, *\right) \doteq \bar{N}
\end{aligned}
$$

which agrees with $F$ over $M^{L-1}$. Moreover, $F^{\prime \prime}$ can be taken cellular.

Remark 3.6. This result, as well as Theorem 3.3, is reminiscent of the following result of J.H.C. Whitehead on simple homotopy types: If $A$ and $B$ are finite CWcomplexes of dimension $n$ with the same $(n-1)$-type and if $f: A \rightarrow B$ realises an $(n-1)$-homotopy equivalence, then there exist $p, q \in \mathbb{N}$ and a simple homotopy equivalence $A \vee\left(S^{n}\right)^{\vee p} \rightarrow B \vee\left(S^{n}\right)^{\vee q}$. In fact, this simple equivalence can be chosen so that it agrees with $f$ over $A^{n-1}$. This is Theorem 14 of [39].

Proof of Theorem 3.5. Consider the filtered space

$$
P=\left(P, P_{L}, P_{L-1}, \ldots, P_{0}=*\right),
$$

where $P$ is the reduced mapping cylinder of $F: M \rightarrow N$ (which has a natural $\mathrm{CW}$ decomposition), and $P_{k}$ is the reduced mapping cylinder of $F^{k}=F_{\mid M^{k}}: M^{k} \rightarrow N^{k}$, for $k=1, \ldots, L-1$. We further define

$$
P_{L}=M^{L} \cup P_{L-1} \cup N^{L}=P^{L} .
$$

Therefore each $P_{k}$ is embedded cellularly in $P_{k+1}$ and $P$, for $k=0, \ldots, L-1$.

We want to prove that there exist filtered homotopy equivalences

$$
\bar{M} \cong P \cong \bar{N}
$$

By Lemma 2.8, there exists a deformation retraction of $P$ onto $M$, say $\rho: P \times I \rightarrow$ $P$, which we can suppose to be cellular, such that the restriction of $\rho$ to $P_{k} \times I$ realises a deformation retraction of $P_{k}$ in $M^{k}$, for $k=0, \ldots, L-1$. Define

$$
r(u)=\rho(u, 1), u \in P .
$$

Therefore $r$ is a cellular map $P \rightarrow M$. 
Let $\left\{e_{1}^{L}, \ldots, e_{n_{L}}^{L}\right\}$ be the set of $L$-cells of $N$. Each of these cells attaches to $N^{L-1}$ along a certain map:

$$
\phi_{i_{L}}^{L}: S_{i_{L}}^{L-1} \doteq \partial\left(e_{i_{L}}^{L}\right) \rightarrow N^{L-1}, i_{L}=1, \ldots, n_{L} .
$$

The CW-complex $P_{L}$ is obtained from $M^{L} \cup P_{L-1}$ by attaching these $L$-cells to it in the obvious way. Notice that $N^{L-1}$ is included in $P_{L-1}$, cellularly.

The deformation retraction $\rho$ from $P$ onto $M$ gives us a homotopy (in $P_{L-1}$ ) connecting $\phi_{i_{L}}^{L}: S_{i_{L}}^{L-1} \rightarrow N^{L-1} \subset P_{L-1}$ with $\left(r \circ \phi_{i_{L}}^{L}\right): S_{i_{L}}^{L-1} \rightarrow M^{L-1} \subset P_{L-1}$, for $i_{L}=1, \ldots, n_{L}$, since the restriction of $\rho$ to $P_{L-1} \times I$ is a deformation retraction from $P_{L-1}$ onto $M^{L-1}$. On the other hand, the restriction $r_{\mid e_{i_{L}}^{L}}$ of $r$ to the $L$-cell $e_{i_{L}}^{L}$ will provide a null homotopy $\left(r \circ \phi_{i_{L}}^{L}\right) \rightarrow *\left(\right.$ in $M^{L}$ ) for $i_{L}=1, \ldots, n_{L}$ (recall that $r$ is cellular, thus $\left.r\left(e_{i_{L}}^{L}\right) \subset M^{L}\right)$. Note that we denote by $*$ both the base point and any function with values in $\{*\}$, and the same for any singleton $\{a\}$. We have proved that each attaching map $\phi_{i_{L}}^{L}: \partial\left(e_{i_{L}}^{L}\right) \rightarrow M^{L} \cup P_{L-1}$, where $i_{L}=1, \ldots, n_{L}$, is null homotopic.

Note that the CW-complex $M^{L}$ is a deformation retract of $M^{L} \cup P_{L-1}$, since $M^{L-1} \subset M^{L}$ is a deformation retract of $P_{L-1}$, and $M^{L-1}=P_{L-1} \cap M^{L}$. In particular the inclusion map $M^{L} \stackrel{i}{\rightarrow} M^{L} \cup P_{L-1}$ is a homotopy equivalence. Therefore, there exist homotopy equivalences

$$
\left(M^{L}, *\right) \vee \bigvee_{i_{L}=1}^{n_{L}}\left(S_{i_{L}}^{L}, *\right) \cong\left(M^{L} \cup P_{L-1}, *\right) \vee \bigvee_{i_{L}=1}^{n_{L}}\left(S_{i_{L}}^{L}, *\right) \cong\left(P_{L}, *\right),
$$

the second one being implied by the fact that the attaching maps $\phi_{i_{L}}^{L}, i_{L}=1, \ldots, n_{L}$ of the $L$-cells of $N$ are null homotopic in $M^{L} \cup P_{L-1}$. Let $G_{L}$ be their composition, thus $G_{L}$ defines a homotopy equivalence

$$
G_{L}:\left(M^{L}, *\right) \vee \bigvee_{i_{L}=1}^{n_{L}}\left(S_{i_{L}}^{L}, *\right) \rightarrow\left(P_{L}, *\right) .
$$

Note that the restriction of $G_{L}$ to $M^{L}$ is (or can be chosen to be) the inclusion map $M^{L} \rightarrow P_{L}$. By Corollary 2.8, the restriction of this inclusion map to $M^{L-1}$ defines a filtered homotopy equivalence $\left(M^{L-1}, \ldots, M^{0}=*\right) \cong\left(P_{L-1}, \ldots, P_{0}=*\right)$. Therefore it follows from Theorem 2.3 that $G_{L}$ is also a filtered homotopy equivalence

$$
G_{L}:\left(M^{L}, M^{L-1}, \ldots, M^{0}=*\right) \vee \bigvee_{i_{L}=1}^{n_{L}}\left(S_{i_{L}}^{L}, *, \ldots, *\right) \rightarrow\left(P_{L}, P_{L-1}, \ldots, P_{0}=*\right)
$$

Given that the restriction of $G_{L}$ to $M^{L}$ is the restriction of the inclusion map $M \rightarrow P$ to $M^{L}$, we can conclude that the map $G_{L}$ extends to a filtered map (which we also call $G_{L}$ )

$$
\begin{aligned}
G_{L}:\left(M, M^{L}, M^{L-1}, \ldots, M^{0}=*\right) & \vee \bigvee_{i_{L}=1}^{n_{L}}\left(S_{i_{L}}^{L}, S_{i_{L}}^{L}, *, \ldots, *\right) \\
& \rightarrow\left(P, P_{L}, P_{L-1}, \ldots, P_{0}=*\right),
\end{aligned}
$$


which when restricted to $M$ is the inclusion map $M \rightarrow P$.

We want to prove that $G_{L}$ extends to a map $G: M \vee\left(D^{L+1}\right)^{\vee n_{L}}=\bar{M} \rightarrow P$. To do so, we need to prove that the restriction of $G_{L}$ to each $S_{i_{L}}^{L} \cong D_{i_{L}}^{L} / \partial D_{i_{L}}^{L}$ is null homotopic.

Let us be more explicit about the construction of $G_{L}$. It is important to remember that the map $G_{L}$ depends explicitly on the null homotopies

$$
H_{i_{L}}: * \rightarrow\left(\phi_{i_{L}}^{L}: \partial\left(e_{i_{L}}^{L}\right) \rightarrow N^{L-1} \subset M^{L} \cup P_{L-1}\right),
$$

which we choose. Here $i_{L}=1, \ldots, n_{L}$. These homotopies are taken in $M^{L} \cup P_{L-1}$. Let us describe each $H_{i_{L}}: \partial\left(e_{i_{L}}^{L}\right) \times I \rightarrow M^{L} \cup P_{L-1}$ in full detail, as follows.

Let $i_{L} \in\left\{1, \ldots, n_{L}\right\}$. Recall $S_{i_{L}}^{L-1} \doteq \partial\left(e_{i_{L}}^{L}\right)$. Define $H_{i_{L}}: S_{i_{L}}^{L-1} \times I \rightarrow M^{L} \cup$ $P_{L-1}$ in the following way: Let $a_{i_{L}}^{L}$ be the central point of the closure $\overline{e_{i_{L}}^{L}} \cong D^{L}$ of $e_{i_{L}}^{L}$. Therefore there exists a path $\gamma_{i_{L}}^{L}$ in $M^{L}$ connecting the base point $*$ with $r\left(a_{i_{L}}^{L}\right)$. When $t \in\left[0, \frac{1}{3}\right] \subset I$, we define $H_{i_{L}}$ directly from $\gamma_{i_{L}}^{L}$, in the obvious way. This yields a homotopy $J_{i_{L}}^{1}: * \rightarrow r\left(a_{i_{L}}^{L}\right)$, in $M^{L}$. Specifically

$$
J_{i_{L}}^{1}(x, t)=\gamma_{i_{L}}^{L}(t), \forall x \in S_{i_{L}}^{L-1}, \forall t \in I .
$$

When $t \in\left[\frac{1}{3}, \frac{2}{3}\right] \subset I$, we consider $H_{i_{L}}$ to be defined in the natural way from the restriction $r_{\mid i_{i_{L}}^{L}}$ of $r$ to $e_{i_{L}}^{L}$. This defines a homotopy $J_{i_{L}}^{2}: r\left(a_{i_{L}}^{L}\right) \rightarrow\left(r \circ \phi_{i_{L}}^{L}\right)$, in $M^{L}$. Explicitly $\left(\right.$ note $\left.\overline{e_{i_{L}}^{L}} \cong D^{L}=[-1,1]^{L}\right)$,

$$
J_{i_{L}}^{2}(x, t)=r_{\mid e_{i_{L}}^{L}}(x t), \forall x \in S_{i_{L}}^{L-1}, \forall t \in I=[0,1] .
$$

Recall that $e_{i_{L}}^{L}$ attaches along $\phi_{i_{L}}^{L}: S_{i_{L}}^{L-1} \rightarrow N^{L-1} \subset M^{L} \cup P_{L-1}$.

Finally, the deformation retraction $\rho: P \times I \rightarrow P$ (of $P$ onto $M$ ) will define a homotopy $J_{i_{L}}^{3}$ (in $P_{L-1}$ ) connecting $\left(r \circ \phi_{i_{L}}^{L}\right): S_{i_{L}}^{L-1} \rightarrow M^{L-1} \subset M^{L} \cup P_{L-1}$ with $\phi_{i_{L}}^{L}: S_{i_{L}}^{L-1} \rightarrow N^{L-1} \subset M^{L} \cup P_{L-1}$. Explicitly,

$$
J_{i_{L}}^{3}(x, t)=\rho\left(\phi_{i_{L}}^{L}(x), 1-t\right), \forall x \in S_{i_{L}}^{L-1}, \forall t \in I .
$$

It is important to remember that the restriction of $\rho$ to $P_{L-1} \times I$ defines a deformation retraction of $P_{L-1}$ in $M^{L-1}$. This is an extremely crucial fact.

We thus define $H_{i_{L}}: * \rightarrow \phi_{i_{L}}^{L}$ as the concatenation

$$
H_{i_{L}}=\left(J_{i_{L}}^{1} J_{i_{L}}^{2} J_{i_{L}}^{3}\right): S_{i_{L}}^{L-1} \times I \rightarrow M^{L} \cup P_{L-1},
$$

for $i_{L}=1, \ldots, n_{L}$.

As we have seen, when restricted to $M^{L}$, the map $G_{L}$ is the restriction of the inclusion map $M \rightarrow P$ to $M^{L}$. For each $i_{L}=1, \ldots, n_{L}$, the restriction $K_{i_{L}}^{L}$ of $G_{L}$ to $S_{i_{L}}^{L}=D_{i_{L}}^{L} / \partial D_{i_{L}}^{L}$ is given, in the obvious way, from ${ }^{4}$ :

1. The homotopy, $H_{i_{L}}: S_{i_{L}}^{L-1} \times I \rightarrow M^{L} \cup P_{L-1}$ (connecting * with the map $\left.\phi_{i_{L}}^{L}: S_{i_{L}}^{L-1} \rightarrow N^{L-1} \subset M^{L} \cup P_{L-1}\right)$, for $\frac{1}{3} \leqslant|x| \leqslant 1, x \in D_{i_{L}}^{L} / \partial D_{i_{L}}^{L}$.

${ }^{4}$ This is the standard homotopy equivalence $A \cup_{f} D^{n} \rightarrow A \cup_{g} D^{n}$, where $A$ is some space, constructed from a homotopy $H: S^{n-1} \times I \rightarrow A$ connecting $f: S^{n-1} \rightarrow A$ with $g: S^{n-1} \rightarrow A$. See for example [23, proof of Proposition 0.18]. 
2. The obvious re-scaling of the restriction $\operatorname{id}_{\mid e_{i_{L}}^{L}}$ of the identity map id: $N \rightarrow N$ to the cell $e_{i_{L}}^{L}$, for $0 \leqslant|x| \leqslant \frac{1}{3}, x \in D_{i_{L}}^{L} / \partial D_{i_{L}}^{L}$. (Recall that the cell $e_{i_{L}}^{L}$ attaches along $\phi_{i_{L}}^{L}: S_{i_{L}}^{L-1} \rightarrow N^{L-1}$.)

We will make precise the construction of $K_{i_{L}}^{L}$ below. The main point is that when $K_{i_{L}}^{L}=G_{L \mid S_{i_{L}}^{L}}$ is considered to be a map $S_{i_{L}}^{L}=D_{i_{L}}^{L} / \partial D_{i_{L}}^{L} \rightarrow P$, it is homotopic (modulo the border) to the map $K^{\prime L}{ }_{i_{L}}$ which is defined, in the natural way, from:

1. the homotopy $J_{i_{L}}^{1} J_{i_{L}}^{2}$ (connecting $*$ with $\left(r \circ \phi_{i_{L}}^{L}\right)$ ), for $\frac{2}{3} \leqslant|x| \leqslant 1$,

2 . the constant homotopy $\left(r \circ \phi_{i_{L}}^{L}\right) \rightarrow\left(r \circ \phi_{i_{L}}^{L}\right)$, for $\frac{1}{3} \leqslant|x| \leqslant \frac{2}{3}$,

3 . the obvious re-scaling of the function $r_{\mid e_{i_{L}}^{L}}$, for $0 \leqslant|x| \leqslant \frac{1}{3}$. (Recall again that $e_{i_{L}}^{L}$ attaches along $\phi_{i_{L}}^{L}: S_{i_{L}}^{L-1} \rightarrow N^{L-1}$.)

Indeed, a homotopy $W_{i_{L}}^{L}: D_{i_{L}}^{L} / \partial D_{i_{L}}^{L} \times I \rightarrow P$ connecting $K_{i_{L}}^{L}$ with $K_{i_{L}}^{L}$ is given by

$$
W_{i_{L}}^{L}(x, t)=\left\{\begin{array}{c}
J_{i_{L}}^{1} J_{i_{L}}^{2}(x /|x|, 3(1-|x|)), \text { if } \frac{2}{3} \leqslant|x| \leqslant 1, \forall t \in I, \\
\rho\left(\phi_{i_{L}}^{L}(x /|x|), t+(3-3 t)\left(|x|-\frac{1}{3}\right)\right) \text { if } \frac{1}{3} \leqslant|x| \leqslant \frac{2}{3}, \forall t \in I, \\
\rho\left(\operatorname{id}_{\mid e_{i_{L}}^{L}}(3 x), t\right), \text { if } 0 \leqslant|x| \leqslant \frac{1}{3}, \forall t \in I .
\end{array}\right.
$$

This also gives an explicit definition of $K_{i_{L}}^{L}(x)=W_{i_{L}}^{L}(x, 0)$ as well as $K_{i_{L}}^{L}(x)=$ $W_{i_{L}}^{L}(x, 1)$, where $x \in D_{i_{L}}^{L} / \partial D_{i_{L}}^{L}$.

The map $K_{i_{L}}^{L}: D_{i_{L}}^{L} / \partial D_{i_{L}}^{L} \rightarrow M^{L} \subset M^{L} \cup P_{L-1}$ is obviously null homotopic. This follows from the same argument that shows that the concatenation of a path with its reverse is null homotopic.

We have, therefore, proved that $G_{L}$ extends to a map

$$
\begin{aligned}
G: \bar{M} \doteq\left(M, M^{L}, M^{L-1}, \ldots, M^{0}=*\right) & \vee \bigvee_{i_{L}=1}^{n_{L}}\left(D_{i_{L}}^{L+1}, S_{i_{L}}^{L}, *, \ldots, *\right) \\
& \rightarrow\left(P, P_{L}, P_{L-1}, \ldots, P_{0}=*\right) .
\end{aligned}
$$

Let us prove that $G$ is a filtered homotopy equivalence. Let $G^{k}$ be the restriction of $G$ to $\bar{M}^{k}$, for $k=0, \ldots, L$. As we have seen, for $k=0, \ldots, L-1$, the map $G^{k}: M^{k}=\bar{M}^{k} \rightarrow P_{k}$ is a homotopy equivalence. By construction, $G^{L}$ is a homotopy equivalence as well. Not considering filtrations, the map $G: \bar{M} \rightarrow P$ is also a homotopy equivalence, since its restriction to $M$ is the inclusion map $M \rightarrow P$, which is a homotopy equivalence. Therefore by Theorem 2.3 it follows that $G$ is a filtered homotopy equivalence.

Repeating the same argument for the inclusion of $N$ in $P$, we can prove that there exists a filtered homotopy equivalence

$$
\begin{aligned}
Q: \bar{N} \doteq\left(N, N^{L}, N^{L-1}, \ldots, N^{0}=*\right) \vee \bigvee_{j_{L}=1}^{m_{L}}\left(D_{j_{L}}^{L+1}, S_{j_{L}}^{L}, *, \ldots, *\right) \\
\quad \rightarrow\left(P, P_{L}, P_{L-1}, \ldots, P_{0}=*\right) .
\end{aligned}
$$


Here $m_{L}$ is the number of cells of $M$ of order $L$. Note that both $G$ and $Q$, when restricted to $N^{L-1}$ and $M^{L-1}$, are the inclusion maps in $P$.

The naturally defined retraction $R: P_{L-1} \rightarrow N^{L-1}=\bar{N}^{L-1}$ (see the discussion following Corollary 2.8) is a filtered homotopy inverse of $Q^{L-1}:\left(N^{L-1}, \ldots, N^{0}\right) \rightarrow$ $\left(P_{L-1}, \ldots, P_{0}\right)$. Therefore there exists a filtered homotopy inverse $R^{\prime}$ of $Q$ extending $R$, by Corollary 2.5. Note that $(R \circ G)_{\mid M^{L-1}}=F_{\mid M^{L-1}}$. In particular there exists a filtered homotopy equivalence

$$
\begin{aligned}
F^{\prime}=R^{\prime} \circ G: \bar{M} & =\left(M, M^{L}, M^{L-1}, \ldots, M^{0}=*\right) \vee \bigvee_{i_{L}=1}^{n_{L}}\left(D_{i_{L}}^{L+1}, S_{i_{L}}^{L}, *, \ldots, *\right) \\
& \rightarrow\left(N, N^{L}, N^{L-1}, \ldots, N^{0}=*\right) \vee \bigvee_{j_{L}=1}^{m_{L}}\left(D_{j_{L}}^{L+1}, S_{j_{L}}^{L}, *, \ldots, *\right)=\bar{N}
\end{aligned}
$$

with $F_{\mid M^{L-1}}^{\prime}=F_{\mid M^{L-1}}$. We now need to prove that $F^{\prime}$ can be chosen to be cellular. By definition, $F_{\mid \bar{M}^{L}}^{\prime}: \bar{M}^{L} \rightarrow \bar{N}^{L}$ is cellular. The map $F^{\prime}$ is homotopic (modulo $\bar{M}^{L}$ ) to a cellular map $F^{\prime \prime}$; thus $F^{\prime \prime}$ is a homotopy equivalence. Since the restriction of $F^{\prime \prime}$ to each $\bar{M}^{k}$ is a homotopy equivalence for $k=0, \ldots, L$, it follows that $F^{\prime \prime}: \bar{M} \rightarrow \bar{N}$ is a filtered homotopy equivalence, by Theorem 2.3. The map $F^{\prime \prime}$ agrees with $F$ over $M^{L-1}=\bar{M}^{L-1}$. This finishes the proof of Theorem 3.5.

\section{On the fundamental crossed complex of a CW-complex}

\subsection{The dependence of $\Pi(M)$ on the cell decomposition of $M$}

Let $M$ be a CW-complex, equipped with its skeletal filtration. As we have mentioned before in Subsection 2.2.4, even though the crossed complex $\Pi(M)$ depends strongly on the cellular decomposition of $M$, J.H.C. Whitehead proved that the homotopy type of $\Pi(M)$ depends only on the homotopy type of $M$; see [38]. See also Remark 4.3, below. The following result (Theorem 4.1) strengthens this substantially. We freely use the results and notation of Subsection 2.2.

If $n \in \mathbb{N}$, define $\mathcal{D}^{n}=\left(\mathcal{D}_{m}^{n}, \partial_{m}\right)$ as the crossed complex, which is the trivial group for dimensions not equal to $n$ or $n-1$, whilst $\mathcal{D}_{n}^{n}=\mathbb{Z}$ and $\mathcal{D}_{n-1}^{n}=\mathbb{Z}$, the border map $\partial_{n}: \mathbb{Z} \rightarrow \mathbb{Z}$ being the identity. For $n=2$, the action of $\mathbb{Z}$ in $\mathbb{Z}$ is defined to be the trivial action. Therefore $\mathcal{D}^{n} \cong \Pi\left(D^{n}\right)$, where the $n$-disk $D^{n}$ is given its natural $\mathrm{CW}$-structure with one 0-cell, one $(n-1)$-cell, and one $n$-cell.

Let $M$ be a CW-complex with a unique 0-cell. Recall that by definition we have that $\Pi_{L}(M)=\Pi_{L}\left(M, M^{L-1}, M^{L-2}, \ldots, M^{0}=*\right)$.

Theorem 4.1. Let $M$ and $N$ be $C W$-complexes, each with a unique 0-cell which we take to be their base points. Give $M$ and $N$ their skeletal filtrations. Suppose that $M$ and $N$ are homotopic as topological spaces. There exists an isomorphism of crossed complexes

$$
\Pi(M) \bigvee_{n=1}^{\infty}\left(\left(\mathcal{D}^{n+1}\right)^{\vee}\left(l_{\{M, N\}}^{M}(n)\right)\right) \cong \Pi(N) \bigvee_{n=1}^{\infty}\left(\left(\mathcal{D}^{n+1}\right)^{\vee}\left(l_{\{M, N\}}^{N}(n)\right)\right)
$$


Moreover, if $L \in \mathbb{N}$ we also have

$$
\Pi_{L}(M) \bigvee_{n=1}^{L-1}\left(\left(\mathcal{D}^{n+1}\right)^{\vee\left(l_{\{M, N\}}^{M}(n)\right)}\right) \cong \Pi_{L}(N) \bigvee_{n=1}^{L-1}\left(\left(\mathcal{D}^{n+1}\right)^{\vee\left(l_{\{M, N\}}^{N}(n)\right)}\right)
$$

The constants $l_{\{M, N\}}^{N}(n)$ and $l_{\{M, N\}}^{M}(n)$, where $n \in \mathbb{N}$, were defined at the beginning of Subsection 3.2. They also make sense, though they may be infinite, if $M$ or $N$ have infinite cells of order $L$, for some $L \in \mathbb{N}$.

Proof. This follows immediately from Theorem 3.3 and (R. Brown and P.J. Higgins's) Corollary 2.22.

One simple consequence of this theorem is a new proof of (J.H.C. Whitehead's) Theorem 2.26:

Corollary 4.2. Let $M$ and $N$ be $C W$-complexes with unique 0-cells. Suppose that they are homotopic as (based) spaces. There exists a homotopy equivalence $\Pi(M) \cong$ $\Pi(N)$. In fact, for any $L \in \mathbb{N}$, the crossed complexes $\Pi_{L}(M)$ and $\Pi_{L}(N)$ are also homotopy equivalent.

Proof. This result is an easy consequence of Corollary 2.31 and the previous theorem. Also needed is the universal property defining free products of crossed complexes, as well as Lemma 2.25.

Remark 4.3. Theorem 4.1 has a broad intersection with Theorem 16 of [39]. Roughly speaking, this result due to J.H.C. Whitehead says that if $f: \mathcal{A} \rightarrow \mathcal{B}$ is a homomorphism of finite, $L$-truncated, totally free crossed complexes inducing an isomorphism on the homotopy and homology groups of $\mathcal{A}$ and $\mathcal{B}$ up to dimension $L-1$, then it follows that there exists a simple homotopy equivalence $f^{\prime}: \mathcal{A}^{\prime} \supset \mathcal{A} \rightarrow \mathcal{B}^{\prime} \supset \mathcal{B}$, which when restricted to $\mathcal{A} \subset \mathcal{A}^{\prime}$ is $f$. Here $\mathcal{A}^{\prime}$ and $\mathcal{B}^{\prime}$ are obtained from $\mathcal{A}$ and $\mathcal{B}$ by making a certain number of free products with $\Pi_{L}\left(S^{L}\right)$, where the $L$-sphere $S^{L}$ is given a cell decomposition with unique cells of order 0 and $L$. The notion of simple homotopy equivalence of totally free crossed complexes is also defined in [39]. It is very similar to the relation between crossed complexes indicated by Theorem 4.1.

\subsection{A rational number valued homotopy invariant $I_{\mathcal{A}}$}

4.2.1. The definition of $I_{\mathcal{A}}$

We now need to restrict our discussion to CW-complexes which have only a finite number of $L$-cells for each $L \in \mathbb{N}$, therefore avoiding infinities. As usual, all CWcomplexes that we consider have unique 0-cells, taken to be their base points.

Definition 4.4. A (reduced) crossed complex $\mathcal{A}=\left(A_{n}\right)$ is called finite if $\mathcal{A}$ is $L$ truncated for some $L$ and, moreover, all groups $A_{n}, n \in \mathbb{N}$ are finite.

Let $\mathcal{A}=\left(A_{n}\right)$ be a finite $L$-truncated crossed complex. Therefore, if $M$ is a CW-complex with a finite number of cells of each dimension $L \in \mathbb{N}$, then the number of morphisms $\Pi(M) \rightarrow \mathcal{A}$ is finite. This follows from Lemma 2.17. Moreover, by Proposition 2.19, there exists a one-to-one correspondence between morphisms $\Pi(M) \rightarrow \mathcal{A}$ and morphisms $\Pi_{L}(M) \rightarrow \mathcal{A}$. 
For a set $A$, denote its cardinality by $\#(A)$. As usual, if $M$ is a CW-complex, we denote the number of cells of $M$ of dimension $n$ by $l_{n}^{M}$.

Theorem 4.5. Let $M$ be a $C W$-complex with a unique 0-cell, such that $M$ as only a finite number of cells in each dimension (or, alternatively, up to dimension $L$ ). Also let $\mathcal{A}=\left(A_{n}\right)$ be an L-truncated finite crossed complex. Define

$$
\begin{aligned}
I_{\mathcal{A}}(M) & =\#(\operatorname{Hom}(\Pi(M), \mathcal{A})) \prod_{n=1}^{\infty}\left(\prod_{m=1}^{\infty} \#\left(A_{m+n}\right)^{l_{m}^{M}}\right)^{(-1)^{n}} \\
& =\#\left(\operatorname{Hom}\left(\Pi_{L}(M), \mathcal{A}\right)\right) \prod_{n=1}^{\infty}\left(\prod_{m=1}^{\infty} \#\left(A_{m+n}\right)^{l_{m}^{M}}\right)^{(-1)^{n}}
\end{aligned}
$$

Then $I_{\mathcal{A}}(M)$ does not depend on the $C W$-decomposition of $M$, and it is a homotopy invariant of $M$.

Remark 4.6. Note that Lemma 2.17 ensures that $I_{\mathcal{A}}(M)$ is, in principle, calculable, in a combinatorial way.

Theorem 4.5 is a consequence of the following lemma, easy to prove:

Lemma 4.7. Let $\mathcal{A}=\left(A_{n}\right)$ be a crossed complex. There exists a one-to-one correspondence between morphisms $\mathcal{D}^{n} \rightarrow \mathcal{A}$ and elements of $A_{n}$, where $n \in \mathbb{N}$.

Recall $\mathcal{D}^{n}=\Pi\left(D^{n}\right)$, where $D^{n}$ is provided with its natural cell decomposition with unique cells of order $0,(n-1)$ and $n$.

Proof of Theorem 4.5. Let $M$ and $N$ be homotopic cellular spaces. Give $M$ and $N$ CW-decompositions with a unique 0-cell, and such that $M$ and $N$ have only a finite number of $L$-cells for each $L \in \mathbb{N}$. By Theorem 4.1 we can conclude:

$$
\begin{aligned}
\# \operatorname{Hom}\left(\Pi(M) \bigvee_{n=1}^{\infty}\left(\left(\mathcal{D}^{n+1}\right)^{\vee}\left(l_{\{M, N\}}^{M}(n)\right)\right), \mathcal{A}\right) \\
=\# \operatorname{Hom}\left(\Pi ( N ) \bigvee _ { n = 1 } ^ { \infty } \left(\left(\mathcal{D}^{n+1}\right)^{\left.\left.\vee\left(l_{\{M, N\}}^{N}(n)\right)\right), \mathcal{A}\right) .}\right.\right.
\end{aligned}
$$

Therefore, from the universal property defining free products of crossed complexes, as well as the previous lemma, it follows that

$$
\begin{aligned}
\#(\operatorname{Hom}(\Pi(M), \mathcal{A})) \prod_{n=1}^{\infty} \#\left(A_{n+1}\right)^{l_{\{M, N\}}^{M}(n)} & \\
& =\#(\operatorname{Hom}(\Pi(N), \mathcal{A})) \prod_{n=1}^{\infty} \#\left(A_{n+1}\right)^{l_{\{M, N\}}^{N}(n)} .
\end{aligned}
$$

The result follows from some straightforward algebra. 


\subsubsection{Geometric interpretation of $I_{\mathcal{A}}$}

The results described in Subsections 2.2.4, 2.2.6 and 2.2.7, as well as the notation introduced, will be used actively.

Let $M$ be a path-connected space. Suppose that $M$ has only a finite number of non-trivial homotopy groups, all of which are finite. Cellular spaces of this type are studied in $[\mathbf{1 7}, \mathbf{2 4}]$, for example. They generalise Eilenberg-McLane spaces of finite groups. Define the following "multiplicative Euler Characteristic type" invariant:

$$
X^{\pi}(M)=\prod_{k=1}^{\infty}\left[\#\left(\pi_{k}(M)\right)\right]^{(-1)^{k}} .
$$

In general, if $M$ has a finite number of connected components, define

$$
X^{\pi}(M)=\sum_{M_{0} \in \pi_{0}(M)} \prod_{k=1}^{\infty}\left[\#\left(\pi_{k}\left(M_{0}\right)\right)\right]^{(-1)^{k}},
$$

where the sum is extended to all connected components $M_{0}$ of $M$.

Let $\mathcal{A}$ be a finite (non-reduced) crossed complex as usual, in the sense that $\mathcal{A}$ is $L$-truncated, for some $L \in \mathbb{N}$, and all the groupoids $A_{n}, n \in \mathbb{N}$, are finite. Then the classifying space $|\mathcal{A}|$ of $\mathcal{A}$ is a topological space with a finite number of homotopy groups, all of which are finite, by (R. Brown and P.J. Higgins's) Theorem 2.34. We warn the reader that classifying spaces of crossed complexes do not exhaust all spaces of this type. Let $\mathcal{B}$ be a (reduced) finite crossed complex. If $M$ is a CWcomplex with a finite number of $L$-cells for any $L \in \mathbb{N}$ and a unique 0 -cell, then the (non-reduced) crossed complex $C R S(\Pi(M), \mathcal{B})$ is finite, by Lemma 2.17 and Corollary 2.32. In particular, the quantity

$$
\mathcal{X}^{\pi}(|C R S(\Pi(M), \mathcal{B})|)=\mathcal{X}^{\pi}(\operatorname{TOP}((M, *),(|\mathcal{B}|, *)))
$$

is finite. Recall (R. Brown and P.J. Higgins's) Theorem 2.33.

Lemma 4.8. Let $\mathcal{A}=\left(A_{n}\right)$ be a (non-reduced) finite crossed complex. Let $C$ be the object set of $A_{1}$. As usual, we denote by $A_{n}^{c}$ the set of morphisms of $A_{n}$ with source $c$, where $n \in \mathbb{N}$ and $c \in C$. We have:

$$
X^{\pi}(|\mathcal{A}|)=\sum_{c \in C} \prod_{k=1}^{\infty}\left[\#\left(A_{k}^{c}\right)\right]^{(-1)^{k}} .
$$

Proof. Let $\pi_{0}\left(A_{1}\right)$ be the set of connected components of the groupoid $A_{1}$. There exists a one-to-one correspondence between $\pi_{0}\left(A_{1}\right)$ and the set $\pi_{0}(|\mathcal{A}|)$ of connected components of $|\mathcal{A}|$. Each element of $c \in C$ yields a unique 0 -cell $c$ of $|\mathcal{A}|$. Also let $[c]$ denote the connected component of $A_{1}$ to which $c$ belongs. Recall that $\partial_{n}^{c}$ equals 
Homology, Homotopy and Applications, vol. 9(1), 2007

$\partial_{n}$ restricted to $A_{n}^{c}$, if $c \in C$ and $n \in \mathbb{N}$. We have:

$$
\begin{aligned}
X^{\pi}(|\mathcal{A}|) & =\sum_{M \in \pi_{0}(|\mathcal{A}|)} \prod_{k=1}^{\infty}\left(\#\left(\pi_{k}(M)\right)\right)^{(-1)^{k}} \\
& =\sum_{c \in C} \frac{1}{\#([c])} \prod_{k=1}^{\infty}\left(\#\left(\pi_{k}(|\mathcal{A}|, c)\right)\right)^{(-1)^{k}} \\
& =\sum_{c \in C} \frac{1}{\#\left(\pi_{1}(|\mathcal{A}|, c)\right) \#([c])} \prod_{k=2}^{\infty}\left(\frac{\#\left(\operatorname{ker}\left(\partial_{k}^{c}\right)\right)}{\#\left(\operatorname{im}\left(\partial_{k+1}^{c}\right)\right)}\right)^{(-1)^{k}} \\
& =\sum_{c \in C} \frac{1}{\#\left(\pi_{1}(|\mathcal{A}|, c)\right) \#([c])} \prod_{k=2}^{\infty}\left(\frac{\#\left(\operatorname{ker}\left(\partial_{k}^{c}\right)\right) \#\left(\operatorname{ker}\left(\partial_{k+1}^{c}\right)\right)}{\#\left(A_{k+1}^{c}\right)}\right)^{(-1)^{k}} \\
& =\sum_{c \in C} \frac{\#\left(\operatorname{ker}\left(\partial_{2}^{c}\right)\right)}{\#\left(\pi_{1}(|\mathcal{A}|, c)\right) \#([c])} \prod_{k=2}^{\infty}\left(\#\left(A_{k+1}^{c}\right)\right)^{(-1)^{(k+1)}} .
\end{aligned}
$$

If $c, d \in C$, recall that $A_{1}^{(c, d)}$ denotes the set of morphisms of $A_{1}$ with source $c$ and target $d$. We have $\pi_{1}(|\mathcal{A}|, c)=A_{1}^{(c, c)} / \operatorname{im}\left(\partial_{2}^{c}\right)$. But since $A_{1}$ is a groupoid, it follows that $\#\left(A_{1}^{(c, c)}\right) \#([c])=\#\left(A_{1}^{c}\right), \forall c \in C$. In particular, we have:

$$
\begin{aligned}
X^{\pi}(|\mathcal{A}|) & =\sum_{c \in C} \frac{\#\left(\operatorname{ker}\left(\partial_{2}^{c}\right)\right) \#\left(\operatorname{im}\left(\partial_{2}^{c}\right)\right)}{\#\left(A_{1}^{c}\right)} \prod_{k=2}^{\infty}\left(\#\left(A_{k+1}^{c}\right)\right)^{(-1)^{(k+1)}} \\
& =\sum_{c \in C} \frac{\#\left(A_{2}^{c}\right)}{\#\left(A_{1}^{c}\right)} \prod_{k=2}^{\infty}\left(\#\left(A_{k+1}^{c}\right)\right)^{(-1)^{(k+1)}} \\
& =\sum_{c \in C} \prod_{k=1}^{\infty}\left(\#\left(A_{k}^{c}\right)\right)^{(-1)^{k}} .
\end{aligned}
$$

From Corollary 2.32 we can deduce the following theorem, which at the same time interprets and gives an alternative proof of the existence of the invariant $I_{\mathcal{A}}$, where $\mathcal{A}$ is a finite crossed complex.

Theorem 4.9. Let $\mathcal{A}$ be a finite crossed complex. Let $M$ be a $C W$-complex with a unique 0-cell and a finite number of $L$-cells for each $L \in \mathbb{N}$. We have:

$$
I_{\mathcal{A}}(M)=X^{\pi}(\operatorname{TOP}((M, *),(|\mathcal{A}|, *))) \text {. }
$$

Proof. As we have mentioned before (Theorem 2.33), there exists a map

$$
\psi:|C R S(\Pi(M), \mathcal{A})| \rightarrow \operatorname{TOP}((M, *),(|\mathcal{A}|, *)),
$$

which is a weak homotopy equivalence; a result due to R. Brown and P.J. Higgins, appearing in [13]. Therefore

$$
X^{\pi}(\operatorname{TOP}((M, *),(|\mathcal{A}|, *)))=X^{\pi}(|C R S(\Pi(M), \mathcal{A})|) .
$$

We now need to apply the previous lemma together with Corollary 2.32. 
Remark 4.10. These final results originated from discussions that I had with Ronnie Brown and Tim Porter. In fact, the argument for the simpler case of crossed modules was initiated by them.

\subsubsection{A short discussion and an extension of $I_{\mathcal{A}}$}

Let $\mathcal{A}=\left(A_{n}, \partial_{n}\right)$ be a finite crossed complex. In [40], D. Yetter defined a 3-manifold invariant for any finite crossed module. The invariant $I_{\mathcal{A}}$ explains Yetter's invariant, in the cellular category, and generalises it to crossed complexes. A previous systematic study and upgrading of Yetter's construction was done by T. Porter, and appeared in $[\mathbf{3 0}, \mathbf{3 1}]$. The construction due to M. Mackaay of 4-manifold invariants which appears in [25] is conjecturally related to 3-types, having a further incorporation of their cohomology classes in the manner shown below.

The crossed module case was also studied in $[\mathbf{1 9}, \mathbf{2 0}]$. The first article considered only knot complements, while the second article covered general CW-complexes (for example Subsection 3.1 is almost entirely extracted from [20]). One of the main conclusions of that work was that in the case of knotted surfaces, the invariant $I_{\mathcal{G}}$ (where $\mathcal{G}$ is a finite crossed module) is a non-trivial, very calculable invariant. In fact, [20] contained an algorithm for the calculation of $I_{\mathcal{G}}$ from movie presentations of knotted embedded surfaces in $S^{4}$.

Let $M$ be a finite $\mathrm{CW}$-complex. We can easily describe a morphism $\Pi(M) \rightarrow \mathcal{A}$. Recall Lemma 2.17. Roughly speaking, these morphisms are uniquely specified by their value on the $L$-cells of $M$ as long as we choose for any $L \in \mathbb{N}$ and any $L$-cell $e^{L}$ an element of $\pi_{L-1}\left(M^{L-1}, *\right)$ (defined up to acting by an element of $\pi_{1}\left(M^{1}, *\right)$ ) along which $e^{L}$ attaches to $M^{L-1}$. After these choices are made, there exists a one-to-one correspondence between morphisms $\Pi(M) \rightarrow \mathcal{A}$ and colourings of each $L$-cell of $M$, where $L \in \mathbb{N}$, by an element of $A_{L}$, with the obvious compatibility relations with the boundary maps of $\Pi(M)$ and $\mathcal{A}$. Therefore it is not a difficult task to calculate $I_{\mathcal{A}}(M)$ where $M$ is a $\mathrm{CW}$-complex with a unique 0-cell, and its calculation is of a combinatorial nature, similar to the calculation of the cellular homology groups of $M$. We refer to [20] for some calculations in the crossed module case.

The invariant $I_{\mathcal{A}}$ can be naturally twisted by $n$-dimensional cohomology classes $\omega$ of $|\mathcal{A}|$ (the classifying space of $\mathcal{A}$ ) to an invariant $I_{M}(, \omega)$, as long as we restrict $I_{\mathcal{A}}(, \omega)$ to oriented $n$-dimensional closed manifolds. Indeed, if $\omega \in H^{n}(|A|)$, we can define

$$
\begin{aligned}
I_{\mathcal{A}}(M, \omega)= & \sum_{f \in[(M, *),(|\mathcal{A}|, *)]}\left\langle o_{M}, f^{*}(\omega)\right\rangle \\
& \prod_{k=1}^{\infty} \#\left(\pi_{k}(\operatorname{TOP}((M, *),(|\mathcal{A}|, *)), f)\right)^{(-1)^{k}} \\
= & \sum_{F \in \operatorname{Hom}(\Pi(M), \mathcal{A})}\left\langle o_{M}, F_{g}^{*}(\omega)\right\rangle \prod_{n=1}^{\infty}\left(\prod_{m=1}^{\infty} \#\left(A_{m+n}\right)^{l_{m}^{M}}\right)^{(-1)^{n}},
\end{aligned}
$$

where $o_{M}$ is the orientation class of $M$, and, as usual, $l_{n}^{M}$ denotes the number of cells of $M$ of order $n \in \mathbb{N}$. In addition, if $F \in \operatorname{Hom}(\Pi(M), \mathcal{A})$, then $F_{g}:(M, *) \rightarrow(|A|, *)$ 
denotes a geometric realisation of $F$ which is uniquely defined up to homotopy. See [13]. The algebraic description of the cohomology of the classifying space of crossed modules (particular cases of crossed complexes) appears, for example, in $[18,29]$.

From its definition, the invariant $I_{\mathcal{A}}(, \omega)$ is a generalisation of the DijkgraafWitten invariant of manifolds (see [16]). If $M$ is provided with a triangulation, then $I_{\mathcal{A}}(, \omega)$ can be calculated in a similar form to the Dijkgraaf-Witten, but considering colourings on any simplex of $M$, rather than only on the edges of $M$, at least in the untwisted case. We will consider these issues in a subsequent publication; see $[\mathbf{2 1}]$.

\section{References}

[1] H.J. Baues, Combinatorial Homotopy and 4-Dimensional Complexes. Preface by R. Brown, de Gruyter Expositions in Mathematics, vol. 2, Walter de Gruyter \& Co., Berlin, 1991.

[2] H.J. Baues, Algebraic Homotopy, Cambridge Studies in Advanced Mathematics, vol. 15, Cambridge University Press, Cambridge, 1989.

[3] A.L. Blakers, Some relations between homology and homotopy groups, Ann. of Math. 49(2) (1948), 428-461.

[4] R.A. Brown, Generalized group presentation and formal deformations of CWcomplexes, Trans. Amer. Math. Soc. 334(2) (1992), 519-549.

[5] R. Brown, Groupoids and crossed objects in algebraic topology, Homology, Homotopy Appl. 1 (1999), 1-78 (electronic).

[6] R. Brown, Topology and Groupoids, Booksurge LLC, SC, 2006.

[7] R. Brown, On the second relative homotopy group of an adjunction space: an exposition of a theorem of J.H.C. Whitehead, J. London Math. Soc. (2) 22(1) (1980), 146-152.

[8] R. Brown, Crossed complexes and homotopy groupoids as non commutative tools for higher dimensional local-to-global problems, Galois theory, Hopf algebras, and semiabelian categories, Fields Inst. Commun., vol. 43, Amer. Math. Soc., Providence, RI, 2004, pp. 101-130.

[9] R. Brown and P.J. Higgins, On the connection between the second relative homotopy groups of some related spaces, Proc. London Math. Soc. (3) 36(2) (1978), 193-212.

[10] R. Brown and P.J. Higgins, Colimit theorems for relative homotopy groups, J. Pure Appl. Algebra 22(1) (1981), 11-41.

[11] R. Brown and P.J. Higgins, On the algebra of cubes, J. Pure Appl. Algebra 21(3) (1981), 233-260.

[12] R. Brown and P.J. Higgins, Tensor products and homotopies for $\omega$-groupoids and crossed complexes, J. Pure Appl. Algebra 47(1) (1987), 1-33.

[13] R. Brown and P.J. Higgins, The classifying space of a crossed complex, Math. Proc. Cambridge Philos. Soc. 110(1) (1991), 95-120. 
[14] R. Brown, P.J. Higgins and R. Sivera, Nonabelian Algebraic Topology, Part I (preliminary version).

[15] R. Brown and J. Huebschmann, Identities among relations, Low-dimensional topology (Bangor, 1979), London Math. Soc. Lecture Note Ser. vol. 48, Cambridge Univ. Press, Cambridge-New York, 198, pp. 153-2022.

[16] R. Dijkgraaf and E. Witten, Topological gauge theories and group cohomology, Comm. Math. Phys. 129(2) (1990), 393-429.

[17] G. Ellis, Spaces with finitely many non-trivial homotopy groups all of which are finite, Topology 36(2) (1997), 501-504.

[18] G. Ellis, Homology of 2-types, J. London Math. Soc. (2) 46(1) (1992), 1-27.

[19] J. Faria Martins, Categorical groups, knots and knotted surfaces, to appear in J. Knot Theory Ramifications. Preliminary version math.GT/0502562.

[20] J. Faria Martins, On 2-dimensional homotopy invariants of complements of knotted surfaces, math.GT/0507239.

[21] J. Faria Martins and T. Porter, On Yetter's Invariant and an extension of the Dijkgraaf-Witten Invariant to categorical groups, to appear in Theory Appl. Categ. Preliminary version math.QA/0608484.

[22] M. Gutiérrez and P. Hirschhorn, Free simplicial groups and the second relative homotopy group of an adjunction space, J. Pure Appl. Algebra 39(1-2) (1986), 119-123.

[23] A. Hatcher, Algebraic Topology, Cambridge University Press, Cambridge, 2002 .

[24] J.L. Loday, Spaces with finitely many non-trivial homotopy groups, J. Pure Appl. Algebra 24 (1982), 179-202.

[25] M. Mackaay, Finite groups, spherical 2-categories, and 4-manifold invariants, Adv. Math. 153(2) (2000), 353-390.

[26] J.P. May, A Concise Course in Algebraic Topology, Chicago Lectures in Mathematics, University of Chicago Press, Chicago, IL, 1999.

[27] J. Milnor, On spaces having the homotopy type of CW-complex, Trans. Amer. Math. Soc. 90 1959, 272-280.

[28] J. Milnor, The geometric realization of a semi-simplicial complex, Ann. of Math. (2) 65 (1957), 357-362.

[29] S. Paoli, (Co)homology of crossed modules with coefficients in a $\pi_{1}$-module, Homology, Homotopy Appl. 5(1) (2003), 261-296 (electronic).

[30] T. Porter, Interpretations of Yetter's notion of $G$-coloring, simplicial fibre bundles and non-abelian cohomology, J. Knot Theory Ramifications 5(5) (1996), 687-720.

[31] T. Porter, Topological quantum field theories from homotopy $n$-types, $J$. London Math. Soc. (2) 58(3) (1998), 723-732.

[32] A.P. Tonks, On the Eilenberg-Zilber Theorem for crossed complexes, J. Pure Appl. Algebra 179(1-2) (2003), 199-220. 
[33] A.P. Tonks, Theory and applications of crossed complexes, U.W. Bangor Ph.D. thesis, 1994, available at: http://www.informatics.bangor.ac.uk/ public/mathematics/research/ftp/theses/tonks.ps.gz.

[34] G.W. Whitehead, Elements of Homotopy Theory, Graduate Texts in Mathematics, vol. 61, Springer-Verlag, New York-Berlin, 1978.

[35] J.H.C. Whitehead, On adding relations to homotopy groups, Ann. of Math. (2) 42 (1941), 409-428.

[36] J.H.C. Whitehead, Note on a previous paper entitled "On adding relations to homotopy groups", Ann. of Math. (2) 47 (1946), 806-810.

[37] J.H.C. Whitehead, Combinatorial Homotopy I, Bull. Amer. Math. Soc. 55 (1949), 213-245.

[38] J.H.C. Whitehead, Combinatorial Homotopy II, Bull. Amer. Math. Soc. 55 (1949), 453-496.

[39] J.H.C. Whitehead, Simple homotopy types, Amer. J. Math. 72 (1950), 1-57.

[40] D.N. Yetter, TQFT's from homotopy 2-types, J. Knot Theory Ramifications 2(1) (1993), 113-123.

\author{
João Faria Martins jmartins@math.ist.utl.pt \\ Departamento de Matemática \\ Instituto Superior Técnico (Universidade Técnica de Lisboa) \\ Av. Rovisco Pais, 1049-001 \\ Lisboa, Portugal \\ Departamento de Matemática \\ Universidade Lusófona de Humanidades e Tecnologia \\ Av. do Campo Grande, 376, 1749-024 \\ Lisboa, Portugal
}

This article is available at http://intlpress.com/HHA/v9/n1/a13 